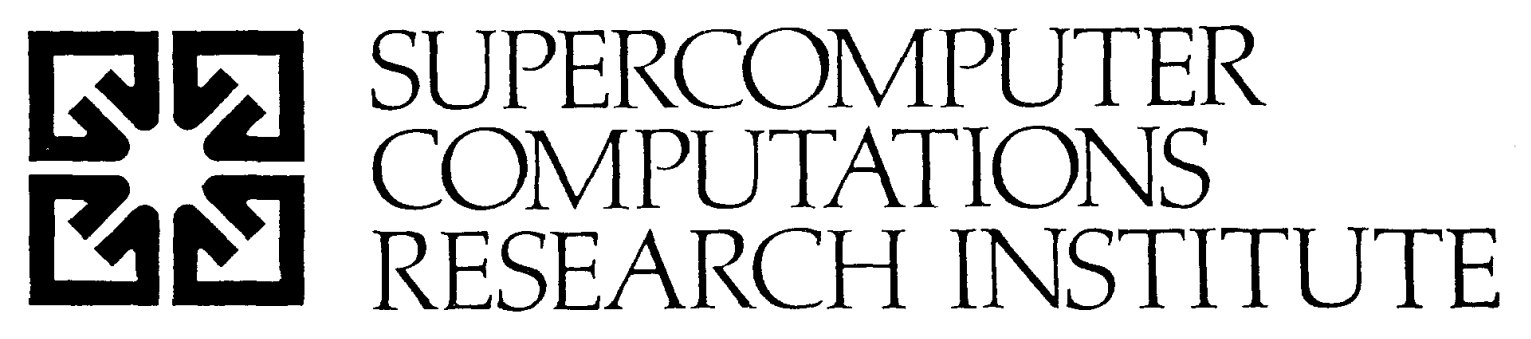

NUMERICAL TRANSFER-MATRIX STUDY

OF A MODEL WITH COMPETING METASTABLE

STATES

by

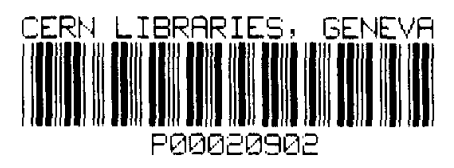

T. Fiig, B. M. Gorman,

P. A. Rikvold and M. A. Novotny

FSU-SCRI-93-160

December 1993

THE FLORIDA STATE UNIVERSITY

TALLAHASSEE, FLORIDA 


\title{
Numerical transfer-matrix study of a model with competing metastable states*
}

\author{
T. Fiig ${ }^{\dagger}$ \\ Supercomputer Computations Research Institute, \\ Florida State University \\ Tallahassee, Florida 32306-4052 \\ and Department of Solid State Physics, \\ Risø National Laboratory \\ DK-4000 Roskilde, Denmark \\ B. M. Gorman and P. A. Rikvold \\ Supercomputer Computations Research Institute, \\ Center for Materials Research and Technology \\ and Department of Physics, \\ Florida State University \\ Tallahassee, Florida 32306-4052 \\ M. A. Novotny \\ Supercomputer Computations Research Institute, \\ Florida State University \\ Tallahassee, Florida 32306-4052
}

December 17, 1993

\footnotetext{
*Submitted to Physical Review E.

† Present address: Department of Solid State Physics, Ris $\varnothing$ National Labozatory, DK-4000 Roskilde, Denmark.
} 


\begin{abstract}
The Blume-Capel model, a three-state lattice-gas model capable of displaying competing metastable states, is investigated in the limit of weak, long-range interactions. The methods used are scalar field theory, a numerical transfer-matrix method, and dynamical Monte Carlo simulations. The equilibrium phase diagram and the spinodal surfaces are obtained by meanfield calculations. The model's Ginzburg-Landau-Wilson Hamiltonian is used to expand the free-energy cost of nucleation near the spinodal surfaces to obtain an analytic continuation of the free-energy density across the first-order phase transition. A recently developed transfer-matrix formalism is applied to the model to obtain complex-valued "constrained" free-energy densities $f_{\alpha}$. For particular eigenvectors of the transfer matrix, the $f_{\alpha}$ exhibit finite-range scaling behavior in agreement with the analytically continued metastable free-energy density. This transfer-matrix approach gives a free-energy cost of nucleation that supports the proportionality relation for the decay rate of the metastable phase $\Gamma \propto\left|\operatorname{Im} f_{\alpha}\right|$, even in cases where two metastable states compete. The picture that emerges from this study is verified by Monte Carlo simulation.
\end{abstract}

PACS Number(s): $64.60 . \mathrm{My}, 64.60 . \mathrm{Qb}, 02.70 . \mathrm{Rw}, 03.50 . \mathrm{Kk}$ 


\section{Introduction}

The investigation of metastable states and their decay through thermally activated nucleation has been the focus of numerous works. (For reviews, see e.g. Refs. study of the analytic properties of the free energy at the condensation point, Langer [3] conjectured that the imaginary part of the free energy $\tilde{F}$ analytically continued from the equilibrium phase across the first-order phase transition may be associated with the decay rate of the metastable phase. A dynamical investigation $[4,5]$ showed for a wide class of models that the decay rate $\Gamma$ may be written in terms of $\operatorname{Im} \bar{F}$ as

$$
\Gamma=\frac{\beta \kappa}{\pi}|\operatorname{Im} \tilde{F}|,
$$

where $\beta$ is the inverse temperature and $\kappa$ is a kinetic prefactor that depends on the dynamics. (We set $k_{\mathrm{B}}=1$ throughout this work.) Subsequently, Binder and collaborators $[6,7,8]$ developed a scaling theory using a nonequilibrium relaxation function to define the metastable states and tested the theory by Monte-Carlo simulation on the two-dimensional Ising model. Schulman and collaborators studied by various methods metastability in the two-dimensional Ising model [9], in the onedimensional Kac model with algebraically decaying interactions [10], in the CurieWeiss model [11], and in a droplet-like "urn" model [12]. In addition, Büttiker and Landauer $[13,14]$ studied nucleation in the over-damped one-dimensional sine-Gordon chain, and Klein and Unger $[15,16]$ studied classical metastability in systems with long-range interactions using a $\phi^{3}$ field theory. Each of these studies supported the validity of Langer's treatment. More recently Gaveau and Schulman [17] determined a rigorous upper bound for the decay rate for a larger class of models than that considered by Langer, and used it not only to explain why Eq. (1) is usually valid, but also provided an example in which Eq. (1) may not appropriately describe the decay rate of the metastable state.

One method for studying metastability that has been used previously is the transfer-matrix approach. Privman and Schulman $[18,19]$ obtained an indication of metastability in their transfer-matrix treatment of the two-dimensional Ising model. Rikvold et al., $[20,21,22]$ studied the stationary properties of metastability in a twostate model with weak, long-range forces, the quasi-one-dimensional Ising (Q1DI) model [23], with a constrained-transfer-matrix (CTM) formalism. This method produces a set of "constrained" free-energy densities $f_{\alpha}$, some of whose imaginary parts have been demonstrated to be related to the metastable decay rate [24]. In particular, strong quantitative agreement was found between the imaginary part of the constrained free-energy density and the analytically continued free-energy density. Recent studies of the two-dimensional Ising model with nearest-neighbor interactions, also using the CTM method $[25,26]$, found that the imaginary part of the constrained free-energy density is consistent with Monte-Carlo estimates of the metastable lifetime [27] and agrees well with field-theoretical droplet-model calculations $[1,3,4,5,28,29]$. 
Our previous studies of metastability $[20,21,22,23,24,25,26,27]$ have focused on systems with one single metastable state. In the present paper we examine a system with two competing metastable states using scalar field theory, the CTM method, and dynamical Monte Carlo simulations. The purpose is two-fold: to test the applicability of the CTM formalism to a system with a more complicated metastability structure and to obtain a clearer understanding of the implications of a recent result of Gaveau and Schulman [17].

This result of Ref. [17] can be summarized as follows. Consider a three-state system with two degenerate metastable phases, $A$ and $B$, and an equilibrium phase $C$, and assume that the reaction path in phase space connects the states only in the following way: $A \rightarrow B \rightarrow C$. According to the Van't Hoff-Arrhenius law [30], the decay rate of $A$ is $\Gamma_{A} \propto \exp \left(-\beta \Delta F_{A \rightarrow B}\right)$, where $\Delta F_{A \rightarrow B}$ is the height of the free-energy barrier on the path $A \rightarrow B$. Gaveau and Schulman argued that the procedure of analytically continuing the free energy for such a system from $C$ into $A$ gives $\operatorname{Im}(\tilde{F}) \propto \exp \left(-\beta \Delta F_{B \rightarrow C}\right)$, where $\Delta F_{B \rightarrow C}$ is defined analogously as above, which when coupled with Eq. (1) does not appropriately describe the decay rate of $A$. Such a discrepancy would give rise to a fundamental question about the physical interpretation of the imaginary part of the constrained free-energy density $\operatorname{Im} f_{\alpha}$ in our CTM formalism. Is $\operatorname{Im} f_{\alpha}$ related to the decay rate in a system with competing metastable states? Based on our analytic and numerical results in this paper, we will argue that $\operatorname{Im} f_{\alpha}$ does indeed give the correct decay rate of $A$.

The system we have chosen is a variant of the Blume-Capel model $[31,32,33]$ with weak, long-range interactions. The Blume-Capel model is an $S=1$ Ising model and is equivalent to a particular three-state lattice-gas model. This model and its generalizations have been studied extensively in the literature, and they have been applied to a variety of different systems (see e.g. Ref. [34] for some chemical applications) and have been studied with different techniques, including mean-field approximations [31, 32, 33], position-space renormalization group [35], transfer-matrix and Monte-Carlo finite-size scaling methods [36], and Monte-Carlo renormalization group [37].

The Hamiltonian for the original ferromagnetic nearest-neighbor Blume-Capel model is given by

$$
\mathcal{H}=-J \sum_{\langle\alpha, \beta\rangle} s_{\alpha} s_{\beta}+D \sum_{\alpha} s_{\alpha}^{2}-H \sum_{\alpha} s_{\alpha}
$$

where the local spin variables $s_{\alpha}$ at site $\alpha$ can take three values, $s_{\alpha}=\{0, \pm 1\}$. The spinspin interaction is ferromagnetic $(J>0), D$ is an applied field that either favors $(D>0)$ or disfavors $(D<0) s_{\alpha}=0$, and $H$ is an applied magnetic field. The indices $\alpha$ and $\beta$ run over a lattice, and the sum $\sum_{\langle\alpha, \beta\rangle}$ runs over nearest neighbors. Unfortunately, the transfer matrix for this model is so complicated that extending it to long-range interactions would be impractical. We therefore introduce a new model, the longrange Blume-Capel model, which we define in Sec. 2 in analogy with the Q1DI model [23]. 
Systems with long-range interactions are often useful to study since they share many characteristics of mean-field models, which are often exactly soluble. Among these characteristics are the presence of metastable and unstable stationary states, which exist for external fields that lie between the first-order phase boundary and a sharp spinodal. (In contrast, in systems with short-range interactions, the spinodal is replaced by a smooth crossover.) In addition, a number of physical systems display behavior characteristic of long-range interactions. Examples include supercooled water [38], superfluid ${ }^{3} \mathrm{He}$ [39], superconductors [40], and long-chain polymer mixtures [41].

The remainder of this paper is organized as follows: In Sec. 2 we define the longrange Blume-Capel model, discuss its zero-temperature phase diagram, and calculate exactly the finite-temperature mean-field spinodal surfaces. The analytic continuation of the free-energy density is considered in Sec. 3. The constrained-transfer-matrix formalism is briefly reviewed in Sec. 4, and its application to the long-range BlumeCapel model is described. Our numerical CTM results are presented in Sec. 5, and the finite-range scaling of the constrained free energy is discussed in Sec. 6. Section 7 contains Monte-Carlo simulation studies of the decay of metastable states, and Sec. 8 contains discussions and conclusions.

\section{Phase Diagram and Spinodal Surfaces}

In this section, we define a Blume-Capel model with weak, long-range interactions, and we calculate the zero-temperature phase diagram and the finite-temperature spinodal surfaces. The system consists of a one-dimensional chain of $L$ layers, each of which contains $N$ spins. The long-range Blume-Capel model is defined by assuming that a given spin $s_{n, i}$, where $i=1, \ldots, L$ and $n=1, \ldots, N$, interacts with each spin in the two adjacent layers with an equal interaction strength $J / N$. We thus replace $J$ with $J / N$ in the first term of the Hamiltonian of Eq. (2), and we allow the sum to range over all spins in adjacent layers. We introduce the following quantities,

$$
\begin{aligned}
m_{i} & =\frac{M_{i}}{N}=\frac{1}{N} \sum_{n=1}^{N} s_{n, i} \\
q_{i} & =\frac{Q_{i}}{N}=\frac{1}{N} \sum_{n=1}^{N} s_{n, i}^{2}
\end{aligned}
$$

which are the magnetization density and the density of nonzero spins in layer $i$, respectively. The Hamiltonian for the long-range Blume-Capel model can then be written as

$$
\mathcal{H}=-J N \sum_{i=1}^{L} m_{i} m_{i+1}+D N \sum_{i=1}^{L} q_{i}-H N \sum_{i=1}^{L} m_{i}
$$

where periodic boundary conditions are imposed, that is $(\forall i) m_{L+i}=m_{i}$ and $q_{L+i}=q_{i}$. 


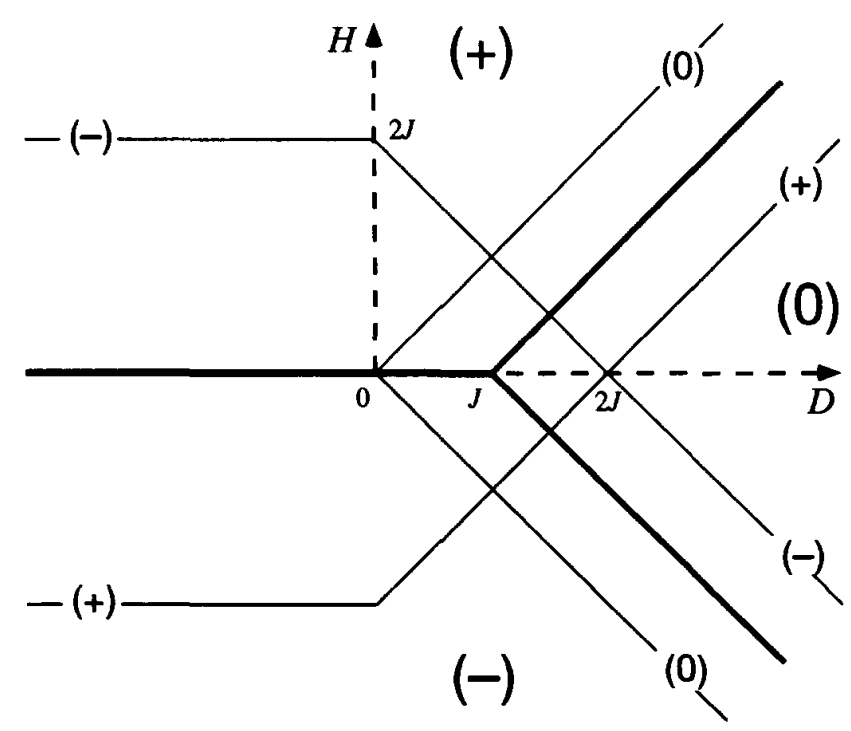

Figure 1: The zero-temperature phase diagram for the long-range Blume-Capel model. The thick solid lines are the first-order equilibrium transition lines, which divide the $(D, H)$ plane into three regions, with stable phases $(0),(+)$, and $(-)$. The thin lines indicate the zero-temperature spinodals for each of the metastable phases.

In the mean-field calculation, we assume that the configurations of the system are translationally invariant, so that $m_{i}=m$ and $q_{i}=q$ for all layers $i=1, \ldots, L$. With this simplification, we can define a mean-field Hamiltonian $\mathcal{H}^{\mathrm{MF}}$ for the energy density as

$$
\mathcal{H}^{\mathrm{MF}}=-J m^{2}+D q-H m .
$$

In the limit $N \rightarrow \infty$, the densities $m$ and $q$ take on continuous values in a domain $\Omega=\{(m, q):|m| \leq q, 0 \leq q \leq 1\}$, which defines an isosceles triangle in $(m, q)$ space.

Before solving this mean-field model in general, we consider its zero-temperature properties. In Fig. 1 the zero-temperature phase diagram is shown. The three stable states are $(m, q)=(0,0),(-1,1)$ and $(1,1)$, which are located at the vertices of the domain $\Omega$. In the following discussion we denote these states as $(0),(-)$, and $(+)$, respectively. The thick solid lines in Fig. 1 are first-order transition lines, which separate the $(D, H)$ plane into three distinct regions, each corresponding to one stable phase, as indicated in the figure. The transition lines are found from a simple energy argument. From the mean-field Hamiltonian of Eq. (5) we can calculate the energy of the three stable phases: $\mathcal{H}^{\mathrm{MF}}(0)=0, \mathcal{H}^{\mathrm{MF}}(+)=D-H-J$, and $\mathcal{H}^{\mathrm{MF}}(-)=D+H-J$. By equating these energies we obtain the transition lines. It follows from this argument that the three states have equal energy at $(D, H, T)=(J, 0,0)$. The three states can also exist as metastable states for certain values of $D$ and $H$ outside the region where they are stable. The reason for this is that as we cross a transition line by changing the fields $D$ and $H$, the previously global minimum of $\mathcal{H}^{\mathrm{MF}}$ remains as 
a local minimum. As we increase the fields further, we eventually reach a spinodal where the local minimum in $\mathcal{H}^{\mathrm{MF}}$ coincides with a saddle point, and the metastable state thus becomes unstable. The spinodal lines at $T=0$ can be calculated by a simple stability analysis, in which we expand $\delta \mathcal{H}$ in terms of $\delta q$ and $\delta m$ around the stationary states and require that $\delta \mathcal{H}=0$. The spinodal lines shown in Fig. 1 are all second-order nonequilibrium transition lines. We obtain for the (0)-spinodal $H= \pm D(D \geq 0)$, for the (-)-spinodal $H=2 J(D \leq 0)$ and $H=2 J-D(D \geq 0)$, and the (+)-spinodal is the reflection of the (-)-spinodal about the $D$-axis.

At finite temperatures the entropic contribution has to be taken into account, so the first-order phase boundaries and spinodal surfaces have to be calculated from the free energy. The mean-field free-energy-density functional can be calculated for a given $m$ and $q$ using the relation $\mathcal{F}^{\mathrm{MF}}=\mathcal{H}^{\mathrm{MF}}-T \mathcal{S}^{\mathrm{MF}}$, where $\mathcal{S}^{\mathrm{MF}}$ is the Boltzmann entropy density of the system, defined by $\mathcal{S}^{\mathrm{MF}}=(1 / N) \ln g(M, Q)$, where $g(M, Q)$ is the multiplicity of spin configurations in a specific layer corresponding to given values of $M$ and $Q$. A simple combinatorial argument yields

$$
g(M, Q)=\left(\begin{array}{c}
N \\
(Q+M) / 2
\end{array}\right)\left(\begin{array}{c}
N-(Q+M) / 2 \\
(Q-M) / 2
\end{array}\right)
$$

Strictly speaking, $\mathcal{F}^{\mathrm{MF}}$ is a functional, but it is related to the thermodynamic freeenergy density $f$ by $f=\min _{(q, m) \in \Omega} \mathcal{F}^{\mathrm{MF}}(m, q)$. In the limit $N \rightarrow \infty, \mathcal{F}^{\mathrm{MF}}$ is given to the leading order of Stirling's approximation as

$$
\begin{aligned}
\mathcal{F}^{\mathrm{MF}}= & -J m^{2}-H m+D q+\frac{1}{2} T[2(1-q) \ln (1-q) \\
& +(q+m) \ln (q+m)+(q-m) \ln (q-m)-2 q \ln 2] .
\end{aligned}
$$

The equilibrium free-energy density is obtained from the global minimum of $\mathcal{F}^{\mathrm{MF}}$ within the domain $\Omega$. By setting the partial derivatives of $\mathcal{F}^{\mathrm{MF}}$ with respect to both $m$ and $q$ equal to zero and defining an effective field $H_{\text {eff }}=H+2 J m$, we obtain the stationarity condition as a pair of coupled equations:

$$
q=\frac{m}{\tanh \left(H_{\text {eff }} / T\right)}
$$

and

$$
\left[1+\frac{1}{4} \exp (2 D / T)\right] q^{2}-2 q+\frac{1}{4} \exp (2 D / T) m^{2}+1=0 .
$$

By combining the two equations, we can write the stationarity condition as an equation only in $m$ :

$$
m=\frac{2 \sinh \left(H_{\mathrm{eff}} / T\right)}{\exp (D / T)+2 \cosh \left(H_{\mathrm{eff}} / T\right)},
$$

Equations (8) and (10) are identical to the expressions found by Blume, et al., [33]. Their simultaneous solution cannot generally be obtained in closed form. A schematic drawing of the mean-field equilibrium phase diagram can be found in Ref. [42]. 
In order to calculate the spinodal surfaces, we require that the local minimum in $\mathcal{F}^{\mathrm{MF}}$ that corresponds to the metastable state is also a point of inflection. This criterion is satisfied if we require, in addition to the vanishing of the partial derivatives of $\mathcal{F}^{\mathrm{MF}}$ with respect to both $m$ and $q$, that the determinant of the Hessian matrix is zero:

$$
\left|\begin{array}{ll}
\mathcal{F}_{m m}^{\mathrm{MF}} & \mathcal{F}_{q m}^{\mathrm{MF}} \\
\mathcal{F}_{\mathrm{mq}}^{\mathrm{MF}} & \mathcal{F}_{\mathrm{qq}}^{\mathrm{MF}}
\end{array}\right|=0
$$

where the subscripts denote partial derivatives with respect to the subscripted variables. Inserting Eq. (7) for $\mathcal{F}^{\mathrm{MF}}$ into Eq. (11), we obtain

$$
2 J m^{2}-2 J q+T=0 .
$$

The spinodal field is obtained by the simultaneous solution of Eqs. (8), (9), and (12), as a relation between $D, H$, and $T$. We start by using Eq. (12) to eliminate $q$ from Eq. (9), which gives

$$
m^{4}(16-4 \eta)+m^{2}[-32+4 \eta+(16-4 \eta) T / J]+16-16 T / J+(4-\eta)(T / J)^{2}=0,
$$

where $\eta=\exp (2 D / T)$. This quadratic equation in $m^{2}$ yields four solutions for $m$,

$$
m= \pm \sqrt{\frac{1}{2}\left[\frac{4 \pm \sqrt{4(1-2 T / J) \eta^{2}+8 \eta T / J}}{4-\eta}-T / J+1\right]}
$$

which we denote $\pm m_{+}$and $\pm m_{-}$. To get the functional relation between the fields, we use Eq. (12) again to eliminate $q$ from Eq. (8). Isolating $H$ from the result gives

$$
H=-2 J m+\frac{T}{2} \ln \left[\frac{2 J m+2 J m^{2}+T}{-2 J m+2 J m^{2}+T}\right] .
$$

By inserting the solution for the magnetization from Eq. (14) into Eq. (15) we obtain the desired expression for the spinodal field $H$ in terms of the field $D$ and temperature $T$.

A three-dimensional plot of the spinodal surface in $(D, H, T)$ space, calculated from the above relation, is shown in Fig. 2. It is symmetric under reflection about the plane $H=0$, due to the symmetry of the Hamiltonian, Eq. (4), under the transformation $\left(s_{n, i}, H\right) \rightarrow\left(-s_{n, i},-H\right)$. Due to the singular behavior of Eq. (15) at $T=0$, the bottom edge of the surface pictured lies at $T / J=1 / 12$. At $T=0$, the spinodal surfaces are anchored to the zero-temperature spinodal lines shown in Fig. 1. Starting from large positive $D$, we have four spinodal sheets corresponding to the four solutions. These are indicated at the right-hand side of the figure. The (0)-spinodal sheets in front and in back merge with the $(-)$ - and (+)-spinodal sheets along the lines $l_{2}$ and $l_{3}$, respectively. Below we obtain analytic expressions for the lines $l_{1}, l_{2}, l_{3}$, and $l_{4}$, including the tricritical point where all four lines meet. As we reduce $D$ past 


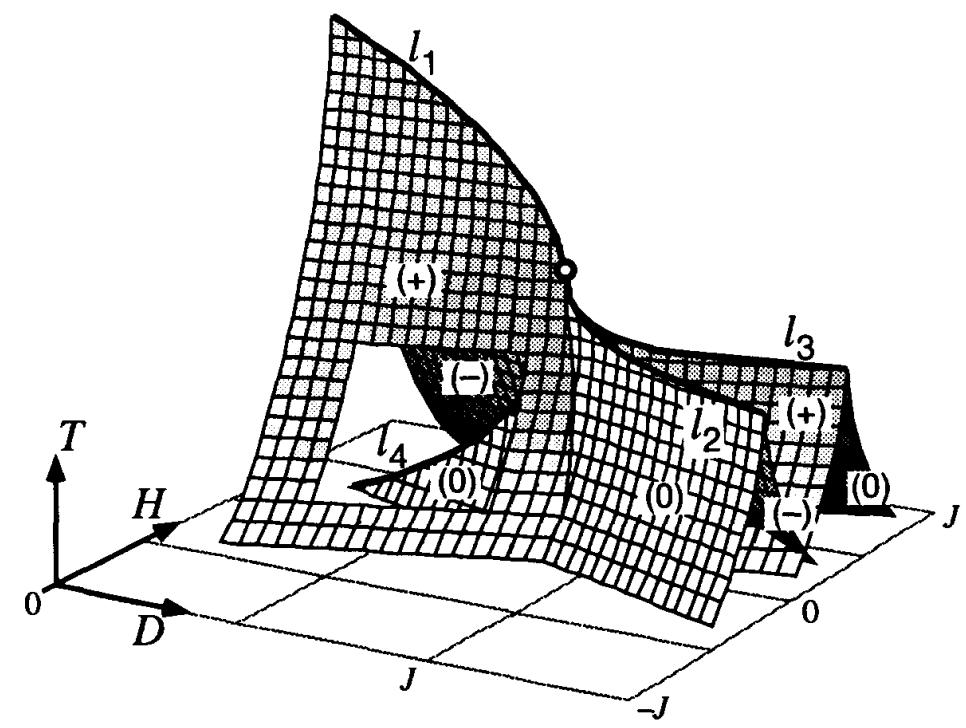

Figure 2: The spinodal surface, shown in $(D, H, T)$ space. The whole surface is symmetric under reflection in the plane $H=0$. Only the portion for $T / J \geq 1 / 12$ is shown, floating above the grid at $T=0$. The two sheets that merge along the line $l_{1}$ are the $(+)$-spinodal in front, and the (-)-spinodal in back. This line of critical points terminates at the tricritical point $\left(D_{t} / J, H_{t} / J, T_{t} / J\right)=\left(\frac{4}{3} \ln 2,0,2 / 3\right)$, which is marked with a circle. The sheets that merge along the line $l_{2}$ are the $(0)$-spinodal in front and the $(-)$-spinodal in back. For the sheets merging along the line $l_{3}$ we have the $(0)$ spinodal in back and the $(+)$-spinodal in front. The two $(0)$-spinodal sheets intersect and cross the $(+)$ - and $(-)$-spinodal surfaces, and merge along the line $l_{4}$. This line connects the point $(D, H, T)=(0,0,0)$ with the tricritical point.

$D=2 J$, the $(+)$ - and (-)-spinodal sheets intersect and cross. The line of intersection becomes $l_{1}$ above the tricritical point. The $(0)$-spinodal sheets pass inside the $(+)$ and $(-)$ sheets and merge along the line $l_{4}$, as can be seen through the rectangular window in the $(+)$ sheet.

Obtaining analytical expressions for the four lines of critical points is quite simple using Eq. (14) for the spinodal magnetizations. The lines are parameterized by the appropriate critical temperature $T_{c}$. The line $l_{1}$ is derived by setting $m_{-}=0$ yielding

$$
l_{1}:\left\{\begin{array}{l}
D_{\mathrm{c}}=T_{\mathrm{c}} \ln \left[\frac{2\left(2 J-T_{\mathrm{c}}\right)}{T_{\mathrm{c}}}\right] \\
H_{\mathrm{c}}=0
\end{array}\right.
$$

for $2 / 3 \leq T_{c} / J \leq 2$. Similarly, $l_{2}$ is obtained from the condition $m_{-}=m_{+}$:

$$
l_{2}:\left\{\begin{array}{l}
D_{\mathrm{c}}=\frac{T_{\mathrm{c}}}{2} \ln \left[\frac{8 T_{\mathrm{c}}}{2 T_{\mathrm{c}}-J}\right] \\
H_{\mathrm{c}}=-2 J \sqrt{-3 T_{\mathrm{c}} / 2 J+1}+T_{\mathrm{c}} \tanh ^{-1}\left[\frac{\sqrt{-3 T_{\mathrm{c}} / 2 J+1}}{-T_{\mathrm{c}} / J+1}\right]
\end{array}\right.
$$

for $1 / 2 \leq T_{\mathrm{c}} / J \leq 2 / 3$. The line $l_{3}$ is the mirror image of $l_{2}$ about the plane $H=0$. The line $l_{4}$ is determined by setting $m_{-}=0$ as was the case for $l_{1}$. The result for $l_{4}$ is 


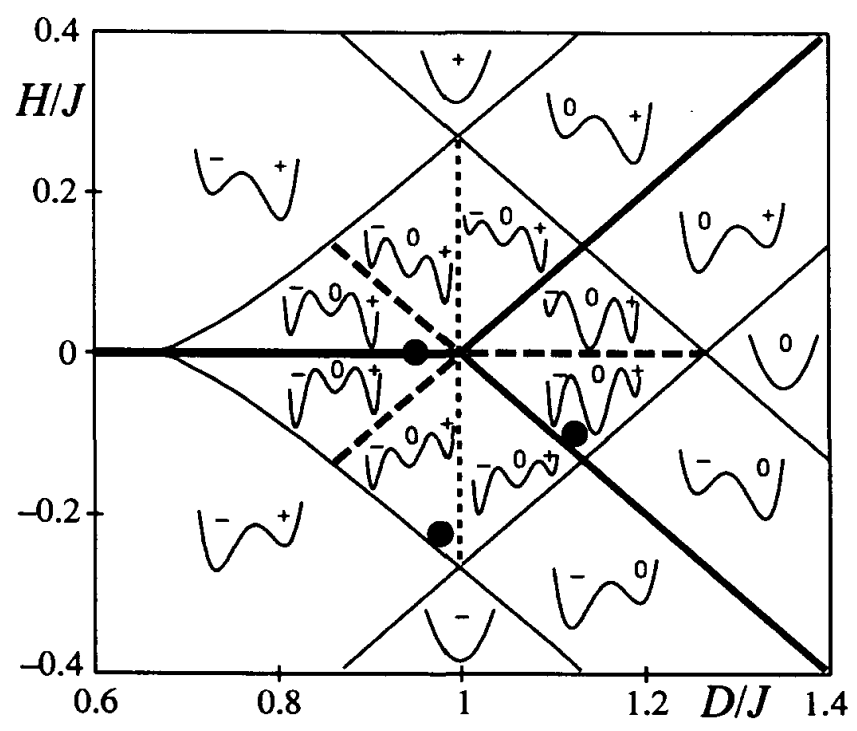

Figure 3: Finite-temperature phase diagram, showing the region of competing metastability, where the spinodal and equilibrium surfaces intersect the plane $T / J=0.25$, well below the tricritical temperature. Thick solid lines represent the firstorder equilibrium transition lines, and thin solid lines are the spinodal lines. The thick dashed lines represent transitions between two metastable states, and the thin dotted vertical line is the locus of equal barrier heights. In each separate region, the freeenergy-density functionals are drawn schematically along the mean-field reaction path. The global minimum corresponds to the equilibrium phase, and local minima represent metastable states. Solid dots indicate parameter sets for which dynamical Monte Carlo simulations of the decay of metastable states were performed (see Fig. 11).

given by Eq. (16) for $0 \leq T_{c} / J \leq 2 / 3$ and is a continuation of $l_{1}$ below the tricritical point. The magnetizations $m_{\mathrm{c}}$ along the lines of critical points are easily found using Eq. (14). Along $l_{1}$ and $l_{4}$ obviously $m_{\mathrm{c}}=0$, since $H=0$ along these lines, whereas along $l_{2}$ and $l_{3}$ we have $m_{\mathrm{c}}^{2}=1-3 T_{\mathrm{c}} / 2 J$. The tricritical point is found as the intersection of the lines $l_{1}$ and $l_{2}: T_{t} / J=2 / 3, D_{t} / J=\frac{4}{3} \ln 2$ and $H_{t}=0$. These analytic expressions for the critical lines are identical to the results obtained by Blume, et al. [33], which in that study were derived from the Landau expansion of the free energy to sixth order.

In Fig. 3 the region of competing metastability at a temperature $T / J=0.25$ (well below $T_{t}$ ) is shown. The thick solid lines are first-order transition lines, and the thin solid lines are spinodal lines. The figure is similar to the zero-temperature phase diagram in Fig. 1, except for the smaller area of the central diamond-shaped region and the curvature in the (0)-spinodals near $H=0$. The lines separate the $(D, H)$ plane into 17 regions, in each of which we have drawn the free-energy-density functional schematically along the most probable mean-field escape path, or mean-field reaction path, joining the three states. Since this path must go through the saddle points of $\mathcal{F}^{\mathrm{MF}}$, it can be shown from the stationarity condition of Eq. (9) that, for the range of fields shown in the figure, each mean-field reaction path is very close to the 
border of $\Omega$ defined by $q= \pm m$. The global minimum represents the equilibrium phase, whereas the remaining local minima represent metastable states. For the $D$ and $H$ values inside the large central diamond-shaped region, two competing metastable states are present. As we cross a spinodal line, the metastable state associated with that spinodal vanishes. The thick dashed lines indicate where the two competing metastable states become degenerate. The thin dotted line for $H \geq 0$ indicates points where the activation barrier heights for the decays $(-) \rightarrow(0)$ and $(0) \rightarrow(+)$ are equal, and analogously for the dotted line for $H \leq 0$. For $H=0$, the activation barrier heights for decay of the $(0)$ metastable state into the degenerate $(+)$ or $(-)$ equilibrium phases are also equal by symmetry. All lines except the exactly obtained spinodal lines have been calculated numerically from Eq. (7) since they involve finding the extrema of $\mathcal{F}^{\mathrm{MF}}$, which has not been done in closed form.

In order to properly characterize the decay of the metastable phases in the longrange Blume-Capel model, we must relax the assumption that the densities $m_{i}$ and $q_{i}$ are translationally invariant. We will show that many of the characteristics of the mean-field model remain, such as the equilibrium and metastable configurations and the spinodals, but we also show, in the next section, that the activation barrier heights are significantly reduced, due to nucleating fluctuations of finite longitudinal extent not present in the mean-field approximation. We can express the Hamiltonian of Eq. (4) as

$$
\mathcal{H}=-J N \sum_{i=1}^{L} \frac{1}{2}\left(m_{i+1}-m_{i}\right)^{2}+N \sum_{i=1}^{L} \mathcal{H}^{\mathrm{MF}}\left(m_{i}, q_{i}\right) .
$$

The free-energy-density functional is then defined as $\mathcal{F}=(\mathcal{H}-T \mathcal{S}) / N L$, where $\mathcal{S}$ is the Boltzmann entropy for the $L \times N$ system. The stationarity condition for the free-energy-density functional $\mathcal{F}$ is then given by $2 L$ coupled equations:

$$
\begin{aligned}
-J\left(m_{i+1}-2 m_{i}+m_{i-1}\right)+\frac{\partial \mathcal{F}^{\mathrm{MF}}\left(m_{i}, q_{i}\right)}{\partial m_{i}} & =0 \\
\frac{\partial \mathcal{F}^{\mathrm{MF}}\left(m_{i}, q_{i}\right)}{\partial q_{i}} & =0
\end{aligned}
$$

for $i=1, \ldots, L$, which can be represented by Eqs. (8) and (9) if we define $H_{\text {eff }}$ as a local effective field, $H_{\text {eff }}=J\left(m_{i+1}+m_{i-1}\right)$, and replace $m$ with $m_{i}$ elsewhere.

In Refs. [24] and [43] it was shown that a two-state model with long-range interactions in an $L^{d-1} \times \infty$ cylindrical geometry can be mapped to a one-dimensional field theory. We will show that a similar mapping can be applied here. We define functions $m(r)$ and $q(r)$ to be continuous in a dimensionless longitudinal coordinate $r$, and we force $m(r)=m_{i}$ and $q(r)=q_{i}$ at integer values $r=i$. By Taylor's theorem, if $i \leq r \leq i+1$, then $\left(m_{i+1}-m_{i}\right)^{2}=(\nabla m(r))^{2}+O\left(\nabla m \nabla^{2} m\right)$. Therefore, if $m(r)$ does not vary too rapidly, then the free-energy density $\mathcal{F}$ for a configuration described by $\left\{m_{i}, q_{i}\right\}$ is well approximated by the Ginzburg-Landau-Wilson form:

$$
\mathcal{F}=\frac{1}{L} \int_{0}^{L} d r\left[\frac{1}{2} J(\nabla m)^{2}+\mathcal{F}^{\mathrm{MF}}(m(r), q(r))\right] .
$$


The density profile $(m(r), q(r))$ of a stationary solution $(\delta \mathcal{F}=0)$ is given by the EulerLagrange equation:

$$
-J \frac{d^{2} m}{d r^{2}}+\left.\frac{\partial \mathcal{F}^{\mathrm{MF}}}{\partial m}\right|_{m(r), q(r)}=0,
$$

where $(\forall r)(m(r), q(r))$ is restricted to lie within $\Omega$ on the ellipse defined by Eq. (9).

In Ref. [24] it was demonstrated that an equation of the form of Eq. (21) has three types of continuous solutions. For each local minimum $\left(m_{\min }, q_{\min }\right)$ of $\mathcal{F}^{\mathrm{MF}}$, there is a uniform solution $(m(r), q(r))=\left(m_{\min }, q_{\min }\right)$, for which the free-energy density is given by the corresponding mean-field result. In addition, for each local minimum ( $\left.m_{m s}, q_{m s}\right)$ that is not the (unique) global minimum, there exists a solution that represents the lowest-lying saddle point between $\left(m_{\mathrm{ms}}, q_{\mathrm{ms}}\right)$ and a second minimum of equal or lower value. If the second minimum is lower, the solution, viewed as a spatial variation in $m$ and $q$, has the shape of a "droplet" embedded in a sea of the metastable configuration $\left(m_{m s}, q_{m s}\right)$. The configuration $(m, q)$ at the center of the droplet is characterized by the solution to $\mathcal{F}^{\mathrm{MF}}(m, q)=\mathcal{F}^{\mathrm{MF}}\left(m_{\mathrm{ms}}, q_{\mathrm{ms}}\right)$ on the arc of the ellipse defined by Eq. (9) between the two minima. If the second minimum is of equal value, the solution has an "interface" shape, in which the system passes from one minimal configuration to the other. These saddle-point solutions exist if the corresponding metastable minima of $\mathcal{F}^{\mathrm{MF}}$ exist. This means that the spinodals derived earlier in the section, which define the limit of metastability for the mean-field Blume-Capel model, also define the limit of metastability for the long-range model. Other solutions to the Euler-Lagrange equation, which are oscillatory in space, can also exist, but we do not consider them here since their associated free-energy densities are higher than those for the solutions already described.

\section{Analytic Continuation of the Free Energy}

For the long-range Blume-Capel model with $T<2 J\left(T_{c}=2 J\right.$ in the Ising limit $D \rightarrow-\infty)$, the free energy $F$ in the limit $N \rightarrow \infty$ is not everywhere analytic, but rather exhibits discontinuous gradients along the first-order phase boundaries. However, if we vary the fields $D$ and $H$ continuously through the boundary, an analytic continuation $\tilde{f}$ of the free-energy density across the boundary exists as an analogue of the thermodynamic free-energy density, but with the partition function constrained to configurations that do not allow the system to decay into the equilibrium phase. The analytic continuation is given as the minimum of $\mathcal{F}$ that coincides with the thermodynamic free-energy density at the phase boundary, but increases as the fields depart from the boundary. When the fields reach the spinodal surface, the metastable minimum vanishes, and the analytic continuation becomes complex outside the spinodal. The objective of this section is to calculate the leading behavior of $\bar{f}$ near the spinodal surface. In Sec. 6 we will compare the finite-range scaling of $\operatorname{Im} \tilde{f}$ with 
that of the imaginary part of the constrained free-energy density obtained numerically from the constrained-transfer-matrix formalism.

Since the metastable state is translationally invariant, the functional minimization of $\mathcal{F}^{\mathrm{MF}}$ in the space of translationally invariant configurations is sufficient to determine $\tilde{f}$ for the metastable states of the long-range model in the limit $N \rightarrow \infty$. Let subscripts to $\mathcal{F}^{\mathrm{MF}}$ denote partial derivatives with respect to the subscripted variables, evaluated at the spinodal. Let $\delta_{m}=m-m_{\mathbf{s}}, \delta_{q}=q-q_{\mathbf{s}}, \delta H=H-H_{\mathbf{s}}$, and $\delta D=D-D_{\mathrm{s}}$ denote variations away from the spinodal. Holding $D=D_{\mathrm{s}}$ constant and varying $H$, we expand the variation of $\mathcal{F}^{\mathrm{MF}}$ from its spinodal value, $\Delta \mathcal{F}^{\mathrm{MF}} \equiv \mathcal{F}^{\mathrm{MF}}\left(m_{\mathrm{s}}+\delta_{m}, q_{\mathrm{s}}+\delta_{q}\right)-\mathcal{F}^{\mathrm{MF}}\left(m_{\mathrm{s}}, q_{\mathrm{s}}\right)$, to third order:

$$
\begin{aligned}
\Delta \mathcal{F}^{\mathrm{MF}}= & -m_{\mathrm{s}} \delta H-\delta_{m} \delta H+\frac{1}{2}\left(\delta_{m}^{2} \mathcal{F}_{m m}^{\mathrm{MF}}+2 \delta_{m} \delta_{q} \mathcal{F}_{m q}^{\mathrm{MF}}+\delta_{q}^{2} \mathcal{F}_{q q}^{\mathrm{MF}}\right) \\
& +\frac{1}{6}\left(\delta_{m}^{3} \mathcal{F}_{m m m}^{\mathrm{MF}}+3 \delta_{m}^{2} \delta_{q} \mathcal{F}_{m m q}^{\mathrm{MF}}+3 \delta_{m} \delta_{q}^{2} \mathcal{F}_{m q q}^{\mathrm{MF}}+\delta_{q}^{3} \mathcal{F}_{q q q}^{\mathrm{MF}}\right)+O\left(\delta^{4}\right)
\end{aligned}
$$

where we have shown only those derivatives that are not identically zero. (Since $\mathcal{F}^{\mathrm{MF}}$ is stationary at the spinodal, $\mathcal{F}_{m}^{\mathrm{MF}}=\mathcal{F}_{q}^{\mathrm{MF}}=0$, and since the fields couple linearly to the order parameters, all field derivatives other than those shown are identically zero.) Since $D$ couples linearly to $q$, the stationarity condition $\mathcal{F}_{q}^{\mathrm{MF}}=0$ gives an expression for $D$ in terms of $m$ and $q$ that can be expanded to give

$$
\delta D=-\delta_{m} \mathcal{F}_{m q}^{\mathrm{MF}}-\delta_{q} \mathcal{F}_{q q}^{\mathrm{MF}}-\frac{1}{2}\left(\delta_{m}^{2} \mathcal{F}_{m m q}^{\mathrm{MF}}+2 \delta_{m} \delta_{q} \mathcal{F}_{m q q}^{\mathrm{MF}}+\delta_{q}^{2} \mathcal{F}_{q q q}^{\mathrm{MF}}\right)+O\left(\delta^{3}\right)
$$

Since we only consider variation in $H, \delta D=0$, and Eq. (23) can be used to express $\delta_{q}$ in terms of $\delta_{m}$ :

$$
\delta_{q}=-\alpha \delta_{m}-\frac{1}{2 \mathcal{F}_{\mathrm{qq}}^{\mathrm{MF}}}\left(\delta_{m}^{2} \mathcal{F}_{m m q}^{\mathrm{MF}}+2 \delta_{m} \delta_{q} \mathcal{F}_{m q q}^{\mathrm{MF}}+\delta_{q}^{2} \mathcal{F}_{\mathrm{qqq}}^{\mathrm{MF}}\right)+O\left(\delta^{3}\right)
$$

where $\alpha \equiv \mathcal{F}_{m m}^{\mathrm{MF}} / \mathcal{F}_{m q}^{\mathrm{MF}}=\mathcal{F}_{m q}^{\mathrm{MF}} / \mathcal{F}_{\mathrm{qq}}^{\mathrm{MF}}$, with the second equality following from the condition for inflection at the spinodal, given by Eq. (11). This expansion and those that follow are valid if $\alpha$ is not singular. Analytic calculation of the derivatives using Eq. (7) shows that $\alpha$ is indeed regular. An expansion for $\delta H$ analogous to Eq. (23) is obtained using the linear coupling of $H$ to $m$ and the stationarity condition $\mathcal{F}_{m}^{\mathrm{MF}}=0$ at the spinodal. We get:

$$
\begin{aligned}
\delta H & =\delta_{m} \mathcal{F}_{m m}^{\mathrm{MF}}+\delta_{q} \mathcal{F}_{m q}^{\mathrm{MF}}+\frac{1}{2}\left(\delta_{m}^{2} \mathcal{F}_{m m m}^{\mathrm{MF}}+2 \delta_{m} \delta_{q} \mathcal{F}_{m m q}^{\mathrm{MF}}+\delta_{q}^{2} \mathcal{F}_{m q q}^{\mathrm{MF}}\right)+O\left(\delta^{3}\right) \\
& =\frac{1}{2} \gamma \delta_{m}^{2}+O\left(\delta^{3}\right),
\end{aligned}
$$

where $\gamma \equiv \mathcal{F}_{m m m}^{\mathrm{MF}}-3 \alpha \mathcal{F}_{m m q}^{\mathrm{MF}}+3 \alpha^{2} \mathcal{F}_{m q q}^{\mathrm{MF}}-\alpha^{3} \mathcal{F}_{q q q}^{\mathrm{MF}}$, and we have used Eq. (24). Substituting the expansions for $\delta_{q}$ and $\delta H$ into Eq. (22) we obtain

$$
\Delta \mathcal{F}^{\mathrm{MF}}=-m_{\mathrm{s}} \delta H-\frac{1}{3} \gamma \delta_{m}^{3}+O\left(\delta^{4}\right) .
$$

By inserting the expression for $\delta_{m}$ in terms of $\delta H$ from Eq. (25), we obtain

$$
\Delta \mathcal{F}^{\mathrm{MF}}=-m_{\mathrm{s}} \delta H-\frac{2 \sqrt{2}}{3} \gamma^{-1 / 2}(\delta H)^{3 / 2}+O\left(\delta H^{2}\right) .
$$


At this point we note that the only assumptions made in the derivation of Eq. (27) are that the two fields couple linearly to their respective order parameters, and that a free-energy-density functional is well-defined in terms of the temperature, fields, and order-parameters. This result is therefore not particular to our model, but rather more general.

We now apply Eq. (27) to our long-range Blume-Capel model. Inserting $\mathcal{F}^{\mathrm{MF}}$ from Eq. (7) and using the condition for inflection at the spinodal, given by Eq. (12), we can express $\gamma$ analytically in terms of $m_{\mathrm{s}}$ and $T$ :

$$
\gamma=\frac{8 m_{\mathrm{s}} J^{2}}{T}\left(m_{\mathrm{s}}^{2}-1+\frac{3 T}{2 J}\right)
$$

Depending on the sign of $m_{\mathrm{s}},\left(m_{\mathrm{s}}^{2}-1+3 T / 2 J\right)$, and $\delta H$, the value of $\Delta \mathcal{F}^{\mathrm{MF}}$ might be real or complex. For the (0)-spinodal surfaces, the equation for the critical magnetization along the lines $l_{2}$ and $l_{3}$ is $m_{\mathrm{c}}^{2}-1+3 T / 2 J=0$ as discussed below Eq. (17); hence for these surfaces,

$$
m_{\mathrm{s}}^{2}-1+\frac{3 T}{2 J}<m_{\mathrm{c}}^{2}-1+\frac{3 T}{2 J}=0 .
$$

Thus there are two cases. If $m_{\mathrm{b}} \delta H<0$, we are inside the (0)-spinodal, and $\Delta \mathcal{F}^{\mathrm{MF}}$ is real, whereas if $m_{\mathbf{s}} \delta H>0$, we are outside the spinodal, and $\Delta \mathcal{F}^{\mathrm{MF}}$ is complex. (Referring to Fig. 2, $m_{s}>0$ for the (0)-spinodal sheet in front, and $m_{\mathrm{s}}<0$ for the (0)spinodal sheet in back.) For the (+)- and (-)-spinodals we can use Eq. (14) for the explicit form of $m_{-}^{2}$ to obtain

$$
\begin{aligned}
m_{\mathrm{s}}^{2}-1+\frac{3 T}{2 J} & =-\frac{1}{2}+\frac{T}{J}+\frac{1}{2} \frac{4-\eta \sqrt{1+2 T(4-\eta) / \eta J}}{4-\eta} \\
& \geq \frac{T}{J}-\left|\frac{1}{2} \frac{\eta}{4-\eta}\left(1-\sqrt{1+2 T \frac{4-\eta}{\eta} J}\right)\right| \\
& \geq \frac{T}{2 J}>0
\end{aligned}
$$

where we have used the inequality $\left|x^{-1}(1-\sqrt{1+a x})\right| \leq|a / 2|$. Therefore, if $m_{\mathrm{s}} \delta H>0$, we are inside the spinodal, whereas if $m_{\mathrm{s}} \delta H<0$, we are outside. (Referring to Fig. 2, $m_{\mathbf{s}}<0$ for the (-)-spinodal sheet, and $m_{\mathrm{a}}>0$ for the ( +$)$-spinodal sheet.) The results for the analytically continued free-energy density are summarized as follows:

$$
\Delta \mathcal{F}^{\mathrm{MF}} \approx \begin{cases}-m_{8} \delta H-\frac{2 \sqrt{2}}{3}|\gamma|^{-1 / 2}|\delta H|^{3 / 2} & \text { metastable state } \\ -m_{8} \delta H \pm i \frac{2 \sqrt{2}}{3}|\gamma|^{-1 / 2}|\delta H|^{3 / 2} & \text { outside spinodal }\end{cases}
$$

where higher-order terms are neglected. Note that this result verifies that every point on the spinodal surfaces calculated in Sec. 2 is a branch point of $\tilde{f}$. From Eq. (31) 
we see that outside the spinodal sheets $\operatorname{Im} \tilde{f}$ is proportional to $\left|H-H_{\mathbf{s}}\right|^{3 / 2}$. The form of Eq. (31), with $\gamma$ appropriately calculated, is true for any mean-field model with two external fields, provided only that the fields couple linearly to their respective order parameters. In the Ising limit $(D \rightarrow-\infty)$ for this model, Eq. (31) agrees with the analytic continuation for a mean-field Ising ferromagnet [11, 44].

Note that if the fields are between the first-order phase boundary and the spinodal, the analytically continued free energy is real-valued only in the limit of infinite interaction range. However, we are interested in the scaling of the free energy for systems with a long, but finite interaction range. For such systems the continuation is complex, but its imaginary part approaches zero rapidly as $N \rightarrow \infty$. The decay of the metastable state happens via the formation of a nucleating droplet of finite extent. The free-energy cost $\Delta F$ associated with the formation of this droplet is evaluated as the minimum barrier height for all paths in phase space that connect the metastable state to the stable state. Combining Langer's result, Eq. (1), with the Van't Hoff-Arrhenius law [30], we expect the imaginary part of the analytically continued free-energy density to have a "Boltzmann weight" given by

$$
\operatorname{Im} \tilde{f} \propto e^{-\beta \Delta F}
$$

Since the density profile of the nucleating droplet can vary longitudinally, we must relax the assumption of translational invariance to find the free-energy cost of the droplet. Taking the continuum limit used in the previous section, we express $\delta_{m}$ and $\delta_{q}$ as continuous functions of $r$, and we expand the variation of $\mathcal{F}$ from its spinodal value to third order. Using the form of Eq. (20) for $\mathcal{F}$, we have

$$
\begin{aligned}
\Delta \mathcal{F} & =\frac{1}{L} \int_{0}^{L} d r\left[\frac{1}{2} J\left(\nabla \delta_{m}\right)^{2}+\Delta \mathcal{F}^{\mathrm{MF}}(m(r), q(r))\right] \\
& =-m_{8} \delta H+\frac{1}{L} \int_{0}^{L} d r\left[\frac{1}{2} J\left(\nabla \delta_{m}\right)^{2}-\delta_{m} \delta H+\frac{1}{6} \gamma \delta_{m}^{3}+O\left(\delta^{4}\right)\right]
\end{aligned}
$$

where we have used Eq. (24) to replace $\delta_{q}$ in Eq. (22) for $\Delta \mathcal{F}^{\mathrm{MF}}$. The Euler-Lagrange equation corresponding to Eq. (21) is thus

$$
-J \nabla^{2} \delta_{m}-\delta H+\frac{1}{2} \gamma \delta_{m}^{2}+O\left(\delta^{3}\right)=0
$$

Following Ref. [24], we take the position of the droplet core to be $r=0$. The critical droplet is local, so the solution to Eq. (34) must be asymptotically uniform in the limit $L \rightarrow \infty$, that is $\delta_{m}(r) \rightarrow$ const. as $|r| \rightarrow \infty$. We thus have asymptotically

$$
\delta_{m} \rightarrow \delta_{0}=(2 \delta H / \gamma)^{1 / 2}+O(\delta H)
$$

where the sign of $\delta_{m}$ is equal to the sign of $\gamma$. If we define $u(r)=\delta_{m}(r)-\delta_{0}$, then Eq. (34) becomes

$$
-J \nabla^{2} u+\gamma \delta_{0} u+\frac{1}{2} \gamma u^{2}+O\left(\delta^{3}\right)=0
$$


In the limit $|r| \rightarrow \infty$ we may neglect the $u^{2}$-term. We thus obtain

$$
u(r) \sim \exp \left(-r / \xi_{\mathbf{r}}\right)
$$

where

$$
\xi_{\mathbf{r}}=(J / \sqrt{2})^{1 / 2}|\gamma \delta H|^{-1 / 4}+O\left(\delta H^{1 / 4}\right)
$$

is the relaxation length of the droplet. The free-energy cost of the critical droplet is given by $\Delta F=N L\left(\Delta \mathcal{F}-\Delta \mathcal{F}_{\mathrm{ms}}^{\mathrm{MF}}\right)$, where $\Delta \mathcal{F}_{\mathrm{ms}}^{\mathrm{MF}}$ is the result in Eq. (31) for the metastable state. Changing variables to $u$ in Eq. (33), we have

$$
\begin{aligned}
\Delta F & =N \int_{0}^{L} d r\left[\frac{1}{2} J(\nabla u)^{2}+\frac{1}{2} \delta_{0} u^{2}+\frac{1}{6} u^{3}+O\left(u^{4}\right)\right] \\
& =-\frac{1}{12} N \gamma \int_{0}^{L} d r\left[u^{3}+O\left(u^{4}\right)\right]
\end{aligned}
$$

where we have integrated $(\nabla u)^{2}$ by parts and used Eqs. (35) and (36). Taking the limit $L \rightarrow \infty$, we place the explicit solution to Eq. $(36)[14,15,16]$,

$$
u(r)=-3 \delta_{0} \operatorname{sech}^{2}\left(r / 2 \xi_{\mathrm{r}}\right),
$$

into the integral of Eq. (39). Evaluating the integral then gives

$$
\begin{aligned}
\Delta F & =\frac{48 \sqrt{2}}{5} N \xi_{\mathrm{r}}|\gamma|^{-1 / 2}|\delta H|^{3 / 2}\left[1+O\left(\delta H^{1 / 2}\right)\right] \\
& =\frac{48}{5} N(\sqrt{2} J)^{1 / 2}|\gamma|^{-3 / 4}|\delta H|^{5 / 4}+O\left(\delta H^{7 / 4}\right) .
\end{aligned}
$$

In the Ising limit $(D \rightarrow-\infty)$, this result reduces to the free-energy cost of nucleation for the Q1DI model [24]. The first expression in Eq. (41) illustrates the difference in the behavior of the analytically continued free energy between long-range models $[15,16,24]$, for which the length scale of the critical droplet is the field and temperature dependent characteristic length $\xi_{\mathrm{r}} \ll L$, and mean-field models $[11,44]$, for which the only length scale for fluctuations is the length $L$ of the entire system.

\section{Constrained-Transfer-Matrix Formalism}

In the first part of this section, the general method of the constrained-transfermatrix formalism is briefly reviewed. A more extensive discussion is found in Refs. $[20,21,24,25]$. In the second part the formalism is applied to the long-range Blume-Capel model.

As the transfer-matrix method is usually applied to equilibrium systems, it provides a way to obtain standard thermodynamic state functions, such as the internal-energy density $\mathcal{U}$, the free-energy density $f$, and correlation functions [45]. Consider a lattice of $L \times N$ sites, with periodic boundary conditions in the $L$ direction. The lattice is divided into $L$ layers, each containing $N$ sites, and the total 
Hamiltonian is decomposed into a sum of layer Hamiltonian operators $\mathcal{H}^{\text {op }}$ whose form is independent of the layer index:

$$
\mathcal{H}=\sum_{i=1}^{L}\left\langle X_{i}\left|\mathcal{H}^{\text {op }}\right| X_{i+1}\right\rangle
$$

where $\left\langle X_{i}\right|$ and $\left|X_{i+1}\right\rangle$ denote the configuration of layers $i$ and $i+1$, respectively. An explicit expression for $\left\langle X_{i}\left|\mathcal{H}^{\text {op }}\right| X_{i+1}\right\rangle$ in the long-range Blume-Capel case is given in Eq. (52) below. The layer Hamiltonian can be further decomposed into a sum of an interaction part, containing only spin-spin interactions, and a field part, containing only terms proportional to the fields. The transfer matrix $\mathbf{T}$ is defined in terms of its matrix elements in the dual space of configurations of two adjacent layers:

$$
\left\langle X_{i}|\mathbf{T}| X_{i+1}\right\rangle=\exp \left[-\beta\left\langle X_{i}\left|\mathcal{H}^{\text {op }}\right| X_{i+1}\right\rangle\right]
$$

A layer configuration $\left|X_{i}\right\rangle$ can be considered as a direct product of single-spin configurations in that layer: $\left|X_{i}\right\rangle \equiv\left|s_{1, i}\right\rangle\left|s_{2, i}\right\rangle \cdots\left|s_{N, i}\right\rangle$. The transfer matrix for the Blume-Capel model is thus $3^{N} \times 3^{N}$. The partition function for the entire $L \times N$ system is given as $\mathcal{Z}=\operatorname{Tr}\left(T^{L}\right)$, and the free-energy density for the system is obtained from $\mathcal{Z}$ as $f=-(T / N L) \ln \mathcal{Z}$. In the limit $L \rightarrow \infty$, the free-energy density becomes $f=-(T / N) \ln \lambda_{0}$, where $\lambda_{0}$ is the largest eigenvalue of the transfer matrix. By the Perron-Frobenius theorem, $\lambda_{0}$ is positive and nondegenerate, and the corresponding eigenvector $|0\rangle$ can be chosen to have all positive elements $[45,46]$.

In the following discussion we restrict ourselves to symmetric transfer matrices $\mathbf{T}$, and we sort the eigenvalues $\lambda_{\alpha}$ in order of decreasing magnitude, so that $\lambda_{0}>\left|\lambda_{1}\right| \geq \cdots \geq\left|\lambda_{3^{N}-1}\right|$. Constrained transfer matrices $\mathbf{T}_{\alpha}$ are constructed from the eigenvalues $\lambda_{\alpha}$ and the corresponding eigenvectors $|\alpha\rangle$ of the equilibrium transfer matrix $\mathbf{T}$. The idea is to reweight the eigenstates in a simple way so that states near equilibrium are suppressed. The matrix $\mathbf{T}_{\alpha}$ is taken to commute with $\mathbf{T}$ and can be written as

$$
\mathbf{T}_{\alpha}=\sum_{\beta \geq 0}|\beta\rangle f_{\beta}(\alpha)\langle\beta|,
$$

where each $|\beta\rangle$ is an eigenvector of $T$. As discussed in Refs. [20, 21, 24, 25], constrained joint and marginal probability densities are defined in analogy with the equilibrium $(\alpha=0)$ case:

$$
\begin{aligned}
P_{\alpha}\left(x_{i}, x_{i+k}\right) & =\left\langle\alpha \mid x_{i}\right\rangle\left\langle x_{i}\left|\left(\lambda_{\alpha}^{-1} \mathbf{T}_{\alpha}\right)^{|k|}\right| x_{i+k}\right\rangle\left\langle x_{i+k} \mid \alpha\right\rangle \\
P_{\alpha}\left(x_{i}\right) & =\left\langle\alpha \mid x_{i}\right\rangle\left\langle x_{i} \mid \alpha\right\rangle .
\end{aligned}
$$

It was pointed out in Refs. $[9,18,19]$ that the constrained marginal probability densities $P_{\alpha}(x)$ can be interpreted as actual probability densities of singlelayer configurations in a constrained state. In order to ensure that the entire system is characterized by $P_{\alpha}(x)$, the matrix $\mathrm{T}_{\alpha}$ must be chosen so that the 
constrained joint probability densities $P_{\alpha}\left(x, x^{\prime}\right)$ satisfy the following regularity conditions: $(i)$ that $P_{\alpha}(x)$ can be obtained by summing over the configurations of one layer, $P_{\alpha}(x)=\sum_{x^{\prime}} P_{\alpha}\left(x, x^{\prime}\right)$; (ii) that $P_{\alpha}\left(x, x^{\prime}\right)$ is well-defined for $k=0$, $P_{\alpha}\left(x_{i}, x_{i}^{\prime}\right)=\delta_{x_{i}, x_{i}^{\prime}} P_{\alpha}\left(x_{i}\right)$; and (iii) that $P_{\alpha}\left(x, x^{\prime}\right)$ reflects stochastic independence in the limit $|k| \rightarrow \infty, \lim _{|k| \rightarrow \infty} P_{\alpha}\left(x_{i}, x_{i+k}\right)=P_{\alpha}\left(x_{i}\right) P_{\alpha}\left(x_{i+k}\right)$. For a matrix $\mathrm{T}_{\alpha}$ chosen to commute with $\mathbf{T}$, these requirements are satisfied if $\mathbf{T}_{\alpha}$ has the same rank as $\mathbf{T}$, and if its dominant eigenvalue is $\lambda_{\alpha}$. Obviously these conditions do not uniquely determine $T_{\alpha}$. For computational convenience we choose a simple form for $T_{\alpha}$ that satisfies the above conditions:

$$
\mathbf{T}_{\alpha}=\sum_{\beta=0}^{\alpha}|\beta\rangle \frac{\lambda_{\alpha}^{2}}{\lambda_{\beta}}\left\langle\beta\left|+\sum_{\beta=\alpha+1}^{3^{N}-1}\right| \beta\right\rangle \lambda_{\beta}\langle\beta|
$$

if $\lambda_{\alpha} \neq 0$, and all terms are zero if $\lambda_{\alpha}=0$. For $\alpha=0$ it is obvious from Eq. (46) that $\mathbf{T}_{\alpha}$ reduces to the equilibrium transfer matrix.

We are now in a position to define constrained, generalized thermodynamic quantities $[20,21]$. The internal-energy density $\mathcal{U}_{\alpha}$ is given by

$$
\mathcal{U}_{\alpha}=\frac{1}{N} \sum_{X, X^{\prime}}\langle\alpha \mid X\rangle\left[\left\langle X\left|\lambda_{\alpha}^{-1} \mathrm{~T}_{\alpha}\right| X^{\prime}\right\rangle\left\langle X\left|\mathcal{H}_{\mathrm{I}}^{\mathrm{op}}\right| X^{\prime}\right\rangle\right]\left\langle X^{\prime} \mid \alpha\right\rangle
$$

where $\mathcal{H}_{\mathrm{I}}^{\mathrm{op}}$ is the interaction part of the layer Hamiltonian. In the Blume-Capel model we have two fields, $H$ and $D$. Consequently we have two field contributions in addition to the interaction part of the Hamiltonian. The order parameter $\mathcal{M}_{\alpha}$ conjugate to the $H$-field is given by

$$
\mathcal{M}_{\alpha}=\frac{1}{N} \sum_{X}\langle\alpha \mid X\rangle\left\langle X\left|M^{\mathrm{op}}\right| X\right\rangle\langle X \mid \alpha\rangle
$$

where $M^{\mathrm{op}}$ is the magnetization operator acting on the layer configuration $|X\rangle$. Similarly, the order parameter $\mathcal{Q}_{\alpha}$ conjugate to the $D$-field is given by

$$
\mathcal{Q}_{\alpha}=\frac{1}{N} \sum_{X}\langle\alpha \mid X\rangle\left\langle X\left|Q^{\mathbf{o p}}\right| X\right\rangle\langle X \mid \alpha\rangle
$$

where $Q^{\mathrm{op}}$ counts the number of nonzero spins in a layer with configuration $|X\rangle$. The constrained entropy density $\mathcal{S}_{\alpha}$ is defined in analogy with that of a stationary ergodic Markov information source [47] and is given by

$$
\mathcal{S}_{\alpha}=-\frac{1}{N} \sum_{X, X^{\prime}}\langle\alpha \mid X\rangle\left[\left\langle X\left|\lambda_{\alpha}^{-1} \mathbf{T}_{\alpha}\right| X^{\prime}\right\rangle \operatorname{Ln}\left\langle X\left|\lambda_{\alpha}^{-1} \mathbf{T}_{\alpha}\right| X^{\prime}\right\rangle\right]\left\langle X^{\prime} \mid \alpha\right\rangle
$$

where $\operatorname{Ln}$ is the principal branch of the complex logarithm. This constrained entropy density may be complex, since $\mathbf{T}_{\alpha}$ is not a positive matrix in general. To see this, 
note that for $|\alpha\rangle(\alpha \neq 0)$ to be orthogonal to $|0\rangle$, the elements of $|\alpha\rangle$ must be of mixed sign. Since $\lambda_{\alpha}$ is the largest eigenvalue of $\mathbf{T}_{\alpha}$, the largest contribution to $\mathbf{T}_{\alpha}$ is the projection $|\alpha\rangle \lambda_{\alpha}\langle\alpha|$, which must contain negative elements. Therefore, the argument to the principal value of the logarithm ( $\mathrm{Ln}$ ) may be negative. The branch cut is taken along the negative real axis, so that the domain of $\ln (z)$ is $|z|>0,-\pi<\arg (z) \leq \pi$. In analogy with equilibrium thermodynamics the free-energy density associated with the eigenstate $|\alpha\rangle$ is defined by

$$
f_{\alpha}=\mathcal{U}_{\alpha}-H \mathcal{M}_{\alpha}+D \mathcal{Q}_{\alpha}-T \mathcal{S}_{\alpha} .
$$

We will apply the formalism described above to the long-range Blume-Capel model Hamiltonian of Eq. (4). From the definition, Eq. (3), it follows that $M_{i}=\left\langle X_{i}\left|M^{\mathrm{op}}\right| X_{i}\right\rangle$ and $Q_{i}=\left\langle X_{i}\left|Q^{\mathrm{op}}\right| X_{i}\right\rangle$. The layer Hamiltonian matrix element $\left\langle X_{i}\left|\mathcal{H}^{\mathbf{o p}}\right| X_{i+1}\right\rangle$ is written in the symmetrized form

$$
\left\langle X_{i}\left|\mathcal{H}^{\mathrm{op}}\right| X_{i+1}\right\rangle=-\frac{J}{N} M_{i} M_{i+1}+\frac{D}{2}\left(Q_{i}+Q_{i+1}\right)-\frac{H}{2}\left(M_{i}+M_{i+1}\right),
$$

so that we obtain a symmetric transfer matrix from Eq. (43). As can be seen from Eq. (52), the layer Hamiltonian is invariant under all permutations of the individual spins, since it only depends on the total magnetizations, $M_{i}$ and $M_{i+1}$, and the total numbers of nonzero spins, $Q_{i}$ and $Q_{i+1}$. This implies that the transfer matrix can be contracted to a reduced basis set consisting of states $\left|M_{i} Q_{i}\right\rangle$, as described in detail in App. 8. The dimension of the contracted transfer matrix is easily seen to be

$$
\operatorname{dim}=\sum_{Q=0}^{N}(Q+1)=\frac{1}{2}(N+1)(N+2),
$$

and the elements of the contracted transfer matrix are given by

$$
\begin{aligned}
\left\langle M Q|\mathrm{~T}| M^{\prime} Q^{\prime}\right\rangle= & \sqrt{g(M, Q) g\left(M^{\prime}, Q^{\prime}\right)} \\
& \times \exp \left(-\beta\left[-\frac{J}{N} M M^{\prime}+\frac{D}{2}\left(Q+Q^{\prime}\right)-\frac{H}{2}\left(M+M^{\prime}\right)\right]\right)
\end{aligned}
$$

where $g(M, Q)$ is the multiplicity factor given in Eq. (6). In App. 8 it is shown that Eqs. (47), (48), and (49) for $\mathcal{U}_{\alpha}, \mathcal{M}_{\alpha}$, and $\mathcal{Q}_{\alpha}$ remain valid with the following substitutions: $|X\rangle \rightarrow|M Q\rangle$ and $\left|X^{\prime}\right\rangle \rightarrow\left|M^{\prime} Q^{\prime}\right\rangle$. Similarly, sums over layer configurations $X$ and $X^{\prime}$ are contracted to sums over $(M, Q)$ and $\left(M^{\prime}, Q^{\prime}\right)$. The only equation that is modified is the expression for the entropy per site [20]:

$$
\begin{aligned}
\mathcal{S}_{\alpha}= & -\frac{1}{N} \sum_{M Q} \sum_{M^{\prime} Q^{\prime}}\langle\alpha \mid M Q\rangle \\
& \times\left[\left\langle M Q\left|\lambda_{\alpha}^{-1} \mathbf{T}_{\alpha}\right| M^{\prime} Q^{\prime}\right\rangle \operatorname{Ln}\left(\frac{\left\langle M Q\left|\lambda_{\alpha}^{-1} \mathbf{T}_{\alpha}\right| M^{\prime} Q^{\prime}\right\rangle}{\sqrt{g(M, Q) g\left(M^{\prime}, Q^{\prime}\right)}}\right)\right]\left\langle M^{\prime} Q^{\prime} \mid \alpha\right\rangle .
\end{aligned}
$$


In addition to the fact that the transfer matrix $T$ is invariant under the spin permutations, which led to the above contraction, other more complex symmetries may be present. This is the case for the long-range Blume-Capel model since, as we show in App. 8, the rank of the matrix is $(2 N+1)$, which is much smaller than the dimension of the contracted matrix. This means that most of the eigenvalues are zero, and that the matrix can be reduced further by projecting out the null space. This further reduction is important, since in order to define the CTM it suffices to diagonalize the reduced matrix. In the numerical computation the reduction of the matrix size from $(N+1)(N+2) / 2$ to $(2 N+1)$ is obviously a great advantage, because much larger system sizes can be studied. In App. 8 a general method for the decomposition of our transfer matrix is discussed.

\section{Numerical Transfer-Matrix Results}

In this section we discuss the numerical results obtained by applying the CTM formalism. Equation (54) is used to set up an $(N+1)(N+2) / 2$ square symmetric transfer matrix T. As described in detail in App. 8, this matrix is block diagonalized by a unitary transformation STS $^{\mathfrak{t}}$ that preserves its symmetries. The null space is then projected out, leaving a $(2 N+1) \times(2 N+1)$ symmetric matrix which is diagonalized using routines tred2 and tqli from Press, et al., [48]. The bulk of the work was performed in 64-bit precision on an HP9000/735 workstation. In certain cases sufficient accuracy could only be obtained using 128-bit precision on a Cray YMP/432 supercomputer. The numerical data presented here required approximately $700 \mathrm{CPU}$-hours on the workstation and $20 \mathrm{CPU}$-hours on the Cray.

The constrained transfer matrices $T_{\alpha}(\alpha=0, \ldots, 2 N)$ are constructed from the eigenvalues $\lambda_{\alpha}$ and eigenvectors $|\alpha\rangle$ using the definition given by Eq. (46). We back-transform $\mathbf{T}_{\alpha}$ from the eigenspace of the reduced transfer matrix into layerconfiguration space $\{|M Q\rangle\}$ by the inverse transformation $\mathbf{S}^{\mathbf{t}} \mathbf{T}_{\alpha} \mathbf{S}$. Using this form for $\mathbf{T}_{\alpha}$ we calculated the constrained free-energy densities $f_{\alpha}$ from Eqs. (47)-(51). The attainable system sizes are limited both by numerical underflow at low temperatures and high fields and by the CPU time required, which increases as $O\left(N^{3}\right)$. We varied the system size from $N=6$ to $N=24$.

Figure 4 shows the eigenvalue spectrum of the equilibrium transfer matrix $\mathbf{T}$ as a function of $H$ for $N=24$ with $D / J=1.1$ and $T / J=0.25$, well below the mean-field tricritical point. The eigenvalues are displayed as $-(T / J N) \ln \left|\lambda_{\alpha}\right|(\alpha=0, \ldots, 2 N)$, so that the lowest branch is the equilibrium free-energy density. The reduced transfer matrix gives a total of 49 branches. These branches are symmetric with respect to $H=0$, and oscillate as functions of $H$, exhibiting avoided crossings at several points. A closer examination of these avoided crossings in the Ising limit revealed that the gaps go to zero exponentially with $N[23]$ up to approximately the spinodal field. Thus the lines denoted as $(0),(-)$, and $(+)$ (see below) actually consist of several branches. It 


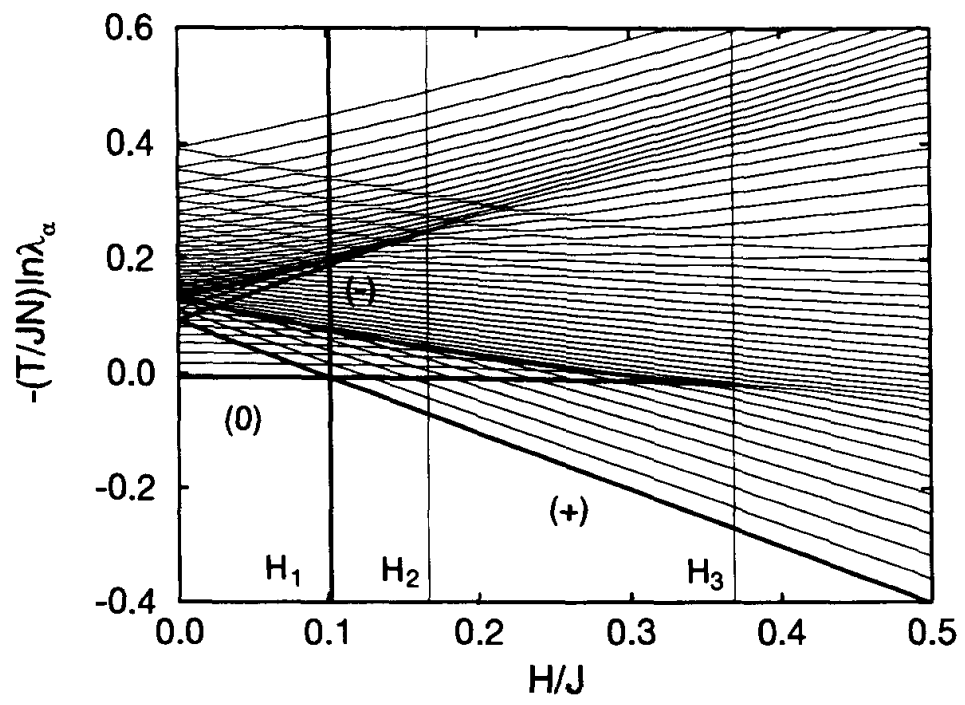

Figure 4: Eigenvalue spectrum for $N=24, D / J=1.1$, and $T / J=0.25$ shown vs. $H / J$ for $0<H / J \leq 0.5$. The spectrum is symmetric upon reflection about $H=0$ and interchange of $(+)$ and $(-)$. The thick vertical line indicates the mean-field first-order transition between the $(0)$ and the $(+)$ state at $H_{1} / J \approx 0.10$, whereas the thin vertical lines mark the (-)-spinodal at $H_{2} / J \approx 0.17$ and the $(0)$-spinodal at $H_{3} / J \approx 0.37$. Also included are bold curves representing the stationary points of the mean-field free-energy-density functional. The virtually straight lines correspond to the stable and metastable states $(0),(+)$, and $(-)$, and the upward-concave curves represent the uniform unstable stationary points. See the text for a detailed description.

was shown by McCraw and Schulman [9] that it is possible to analytically continue the eigenvalues around these crossings.

By drawing a line segment in Fig. 3 at $D / J=1.1$ from $H / J=0$ to $H / J=0.5$ representing the $H$-scan, we can calculate from the mean-field approximation the intersection with both the spinodal and equilibrium lines. The values obtained in this fashion are $H_{1} / J \approx 0.10$ for the first-order equilibrium transition between the $(0)$ state and the $(+)$ state, $H_{2} / J \approx 0.17$ for the (-)-spinodal and $H_{3} / J \approx 0.37$ for the $(0)$ spinodal. These $H$-fields are shown in Fig. 4 as vertical lines. The different branches can be identified by their slopes due to the identity [23]

$$
\frac{T}{J N} \frac{\partial \ln \left|\lambda_{\alpha}\right|}{\partial H}=\mathcal{M}_{\alpha}
$$

Since the (0) state has roughly zero magnetization, the slope of the corresponding branch, by Eq. (56), is nearly zero. Similarly, the $(+)$ and the $(-)$ states have roughly magnetization $m \approx \pm 1$, so the slopes of their corresponding branches are near -1 and +1 , respectively. In Fig. 4 the mean-field solutions for the three local minima in the free-energy functional are shown by bold curves. The $(0)$ state appears as the horizontal line and extends from $H=-H_{3}$ to $H=H_{3}$, the (-) state 


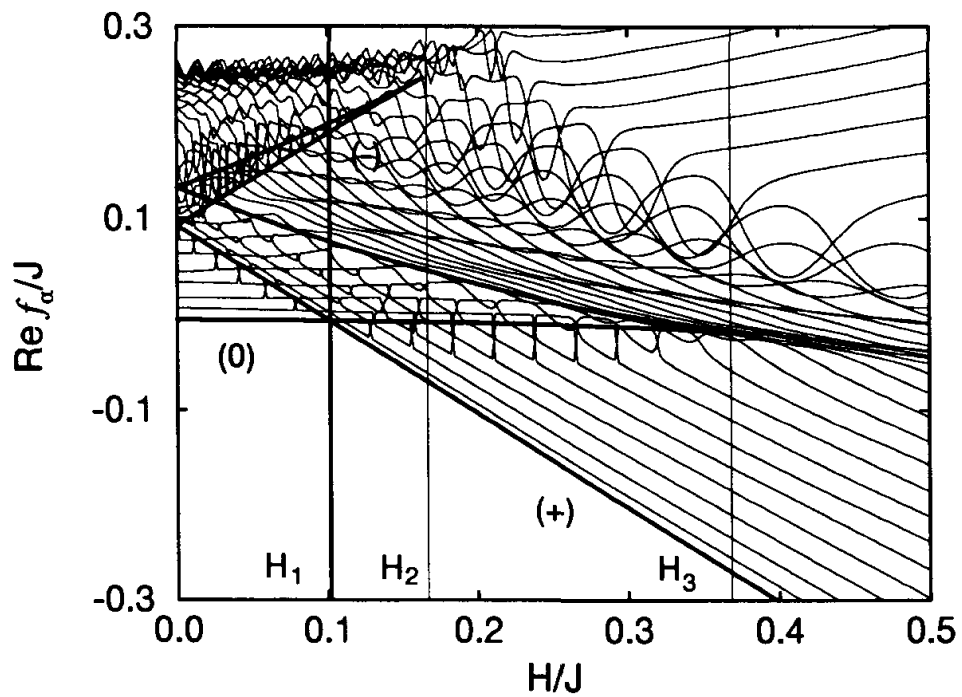

Figure 5: The real parts of the constrained free-energy densities Re $f_{\alpha} / J$ shown $v s$.
$H / J$. The parameters are the same as in Fig. 4 , and the vertical lines and bold curves
have the same meanings. For clarity only 35 of the 49 branches are shown. Note that
the vertical scale is different from Fig. 4 . See the text for a detailed description.

appears as a diagonal line and extends from $H / J=-\infty$ to $H=H_{2}$, and the ( + ) state appears as a diagonal line and extends from $H=-H_{2}$ to $H / J=\infty$. These lines are in such close agreement with the transfer-matrix branches that the branches are completely obscured by them. In addition, the two unstable mean-field solutions are also indicated by bold curves, easily identified by their upward curvature. The two unstable states terminate at the $(-)$-spinodal $\left(H=H_{2}\right)$ and at the $(0)$-spinodal $\left(H=H_{3}\right)$. At the termination points the unstable solutions merge with the metastable states.

The transfer-matrix spectrum in Fig. 4 may be interpreted as follows. For $-H_{1} \leq H \leq H_{1},(0)$ is the lowest-lying branch, and thus represents the stable state, while $(+)$ and $(-)$, which lie above, are metastable. For $H_{1} \leq H \leq H_{2}$ the $(0)$ state has become metastable, while $(+)$ is the stable state, and $(-)$ remains metastable. At $\mathrm{H}=\mathrm{H}_{2}$, the gaps between nearly degenerate eigenvalues vanish far less rapidly with $\mathrm{N}$ than for $\mathrm{H}<\mathrm{H}_{2}$, making the gaps visible in the figure. This corresponds to the crossing of the (-)-spinodal, and hence the disappearance of the metastable (-) state. For $H_{2} \leq H \leq H_{3}$ the $(+)$ state remains stable, while $(0)$ remains metastable until the (0)-spinodal is crossed at $H=H_{3}$. For $H \geq H_{3}$ the (+) state is stable, and no metastable states exist. The transfer-matrix eigenvalues of Fig. 4 thus agree closely with the mean-field picture of metastability discussed in Sec. 2.

The real parts of the constrained free-energy densities, Re $f_{\alpha}$, are shown in Fig. 5. The parameters are the same as in Fig. 4. Out of a total of 49 branches only the 


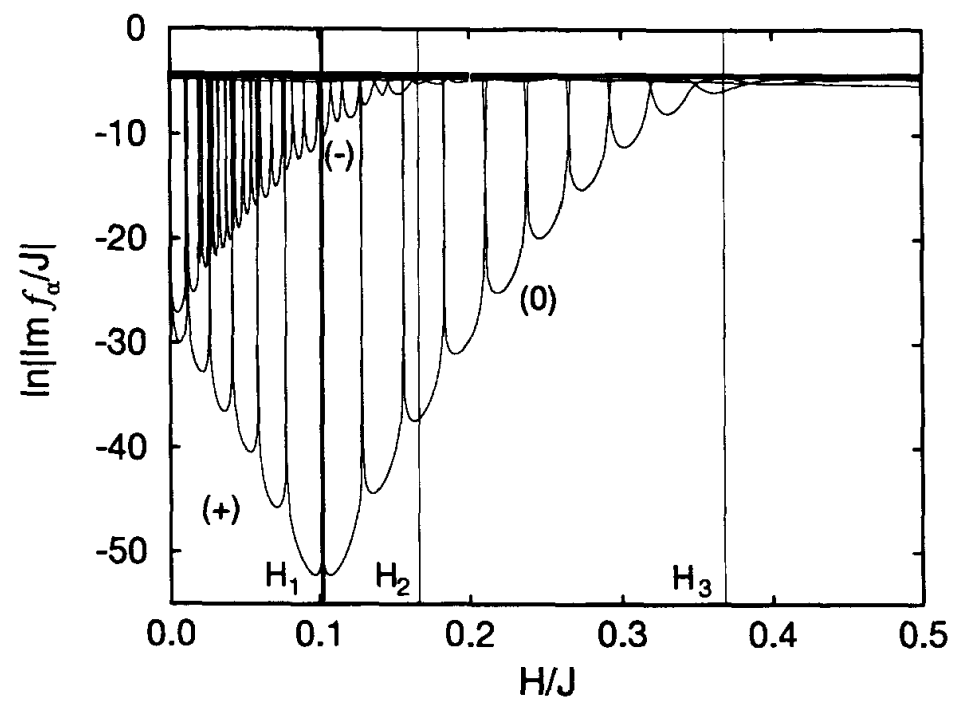

Figure 6: The imaginary parts of the constrained free-energy densities $\left|\operatorname{Im} f_{\alpha} / J\right|$ shown on a logarithmic scale vs. $H / J$. The parameters are the same as in Fig. 4 , and the vertical lines have the same meanings. The envelopes of the lobe structures correspond to different metastable states as indicated. See the text for a detailed description.

35 that correspond to the largest eigenvalues are shown. The remaining branches, which contribute only to the band of unstable states with high free-energy densities and do not extend beyond the range of the branches shown, were removed for clarity. The vertical lines at $H=H_{1}, H_{2}$, and $H_{3}$ have the same meanings as in Fig. 4. The field-dependence of the quantities shown in Figs. 4 and 5 resemble each other closely, and in particular the stable and metastable branches are nearly left unaltered by the reweighting of the eigenvalues. However, the spectrum of the constrained free-energy densities is somewhat compressed compared to the eigenvalue spectrum and contains multilevel crossings. The bold curves in the figure show the five extrema in the meanfield free-energy-density functional corresponding to the $(0),(+)$, and $(-)$ states and the two unstable states, as discussed above. The branches of Re $f_{\alpha}$ representing the stable and metastable branches are again completely obscured by the bold curves, and are thus in excellent agreement with the equilibrium and analytically continued metastable free-energy densities.

The imaginary parts of all the constrained free-energy densities $\left|\operatorname{Im} f_{\alpha}\right|$ are shown on a logarithmic scale versus $H$ in Fig. 6 . The parameters and the vertical lines are the same as in Fig. 4. The most striking features are the extremely small values and the lobe structure of the branches that correspond to the metastable states shown in Figs. 4 and 5. Each lobe corresponds to a different value of $\alpha$, and the crossings of lobes correspond to the avoided crossings in the eigenvalue spectrum. By comparison with the real parts of the constrained free-energy densities, different 


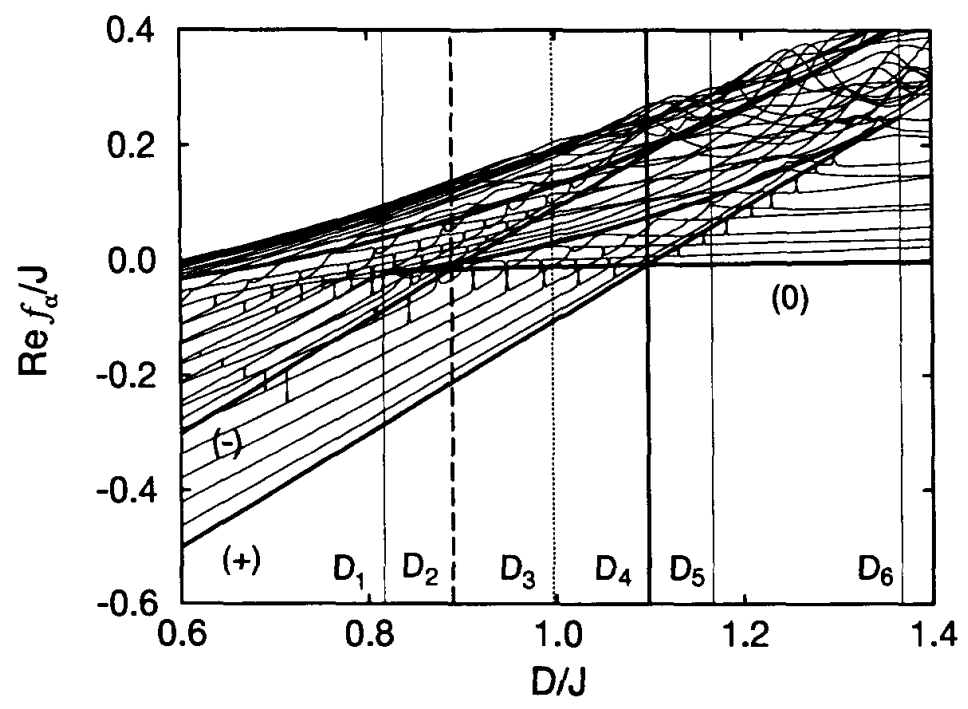

Figure 7: The real parts of the constrained free-energy densities $\operatorname{Re} f_{\alpha} / J$ for $N=18$, $H / J=0.1$, and $T / J=0.25$, shown as functions of $D / J$ for $0.6 \leq D / J \leq 1.4$. The vertical lines indicate the $(0)$-spinodal at $D_{1} / J \approx 0.82$, the exchange of metastable states at $D_{2} / J \approx 0.90$, the point of equal barrier heights at $D_{3} / J \approx 0.99$, the equilibrium transition at $D_{4} / J \approx 1.10$, the $(-)$-spinodal at $D_{5} / J \approx 1.17$, and the $(+)$-spinodal at $D_{6} / J \approx 1.37$. The bold curves have the same meaning as in Figs. 4 and 5 . See the text for a detailed description.

"branches" of the lobe structure can be identified. The set of lobes that decrease in value with increasing $H$ for $0 \leq H \leq H_{1}$ corresponds to the metastable $(t)$ state, whereas the set of lobes that increase in value with increasing $H$ for $H_{1} \leq H \leq H_{3}$ corresponds to the metastable ( 0 ) state. Finally, the set of lobes that start at $H=0$ and terminate at the (-)-spinodal $\left(\mathrm{H}=\mathrm{H}_{2}\right)$ correspond to the metastable (-) state. Since the transfer-matrix eigenvalue spectrum is symmetric about $H=0$, the set of lobes corresponding to the $(+)$ metastable state continues to $H=-\mathrm{H}_{2}$, and the set of lobes corresponding to the $(-)$ metastable state starts at $H=-H_{1}$. The envelopes of these sets of lobes exhibit qualitative behavior that is strikingly similar to that predicted for the analytically continued free-energy densities in that they are exponentially suppressed with increasing $N$ for $|H|$ less than its appropriate spinodal value. In Sec. 6 we will show that this agreement is also quantitative.

The results presented above show the transfer-matrix data as functions of the $H$-field. In Figs. 7 and 8 , the real and imaginary parts of the constrained freeenergy densities are shown as functions of the $D$-field for $0.6 \leq D / J \leq 1.4$ for $N=18$ and $H / J=0.1$ at $T / J=0.25$, giving rise to a total of 37 branches. In analogy with the $H$-scan we draw a line segment in Fig. 3 representing the $D$-scan, thereby obtaining values of $D$ corresponding to the following mean-field transitions: $D_{1} / J \approx 0.82$ for the (0)-spinodal, $D_{2} / J \approx 0.90$ for the exchange of metastable states, $D_{3} / J \approx 0.99$ for the 


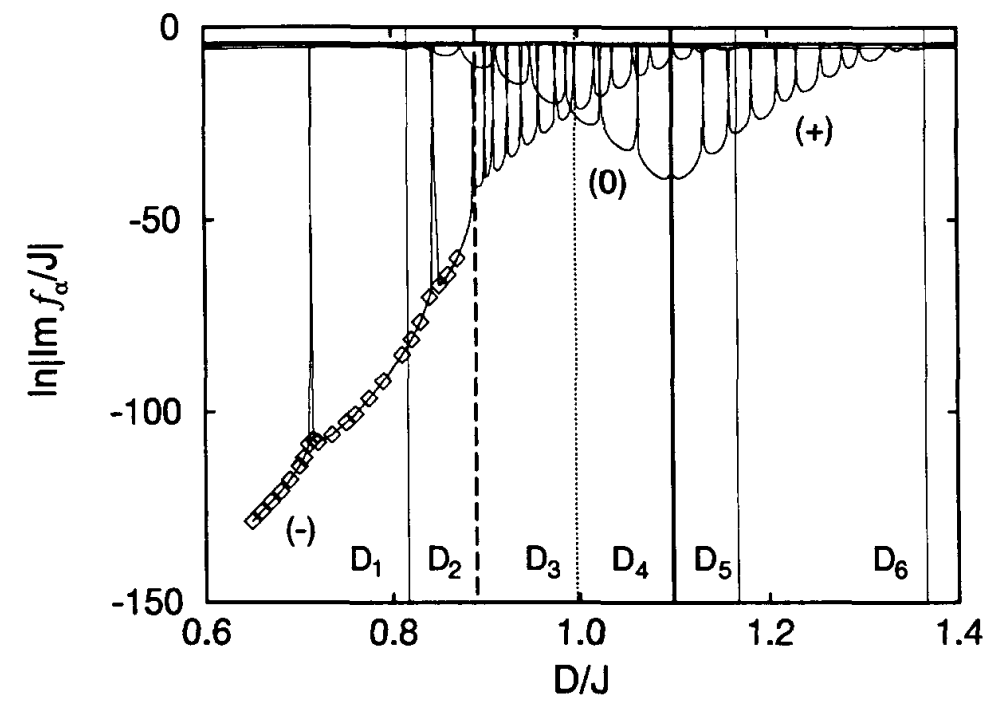

Figure 8: The imaginary parts of the free-energy densities $\left|\operatorname{Im} f_{\alpha} / J\right|$ shown on a logarithmic scale vs. $D / J$. The parameters are the same as in Fig. 7 , and the vertical lines have the same meaning. The envelopes of the lobe structures correspond to different metastable states. Diamonds are data points obtained using extended numerical precision (128 bit). See the text for a detailed description.

barrier heights being equal, $D_{4} / J \approx 1.10$ for the equilibrium transition, $D_{5} / J \approx 1.17$ for the $(-)$-spinodal, and $D_{6} / J \approx 1.37$ for the $(+)$-spinodal. These values are indicated as vertical lines. In Fig. 7 we show $\operatorname{Re} f_{\alpha} / J$ together with bold curves indicating the mean-field results for the stable, metastable and unstable states. The states can again be identified from the slopes of their branches due to the identity

$$
-\frac{T}{J N} \frac{\partial \ln \left|\lambda_{\alpha}\right|}{\partial D}=\mathcal{Q}_{\alpha}
$$

Thus both the $(+)$ and the $(-)$ branches have slopes near unity, while the $(0)$ state has a slope near zero. For $D \leq D_{4}$, the $(+)$ state is stable, while for $D \geq D_{4}$, it remains metastable until the $(+)$-spinodal at $D=D_{6}$. The bold line nearly parallel to the $(+)$ branch is the $(-)$ branch, which terminates at the (-)-spinodal at $D=D_{5}$. The horizontal bold line is the (0) state, which is metastable for $D_{1} \leq D \leq D_{4}$ and stable for $D>D_{4}$. An exchange of $(-)$ and $(0)$ as the lowest-lying metastable state occurs at $D=D_{2}$, as seen by the crossing of the two corresponding bold lines.

The imaginary parts of the constrained free-energy densities $\left|\operatorname{Im} f_{\alpha}\right|$ are shown on a logarithmic scale versus $D$ in Fig. 8. The data shown extend over 55 decades. The solid lines for $\ln \left|\operatorname{Im} f_{\alpha} / J\right| \geq-60$ were calculated with 64 -bit precision on a HP9000/735 workstation. Extended precision (128-bit) was used for the remaining parts. These data points, shown as diamonds, were calculated on a Cray Y-MP/432 supercomputer. The lobe structure is identified as follows. The lobes that increase in value with 
increasing $D$ for $D_{4} \leq D \leq D_{6}$ correspond to the $(+)$ metastable state, whereas those that decrease in value for $D_{1} \leq D \leq D_{4}$ correspond to the $(0)$ metastable state. The remaining lobes for $D \leq D_{5}$ correspond to the $(-)$ metastable state. Again, the qualitative behavior is very similar to the analytically continued free-energy density. Note also that the lobe structures corresponding to the $(-)$ and $(0)$ metastable states intersect at $D \approx D_{3}$, which is approximately equidistant in $H$ from the (-)- and (0)spinodals, as can be seen in Fig. 3. By Eq. (41), the barrier heights $\Delta F_{(-) \rightarrow(0)}$ and $\Delta F_{(0) \rightarrow(+)}$ are therefore equal to leading order in the expansion about the spinodals, just as their mean-field counterparts $\Delta \mathcal{F}^{\mathrm{MF}}$, even though the dependence on $\delta H$ for the mean-field barriers are quite different. This result suggests that $\left|\operatorname{Im} f_{\alpha}\right|$ for the metastable state is related to the free-energy barrier height involved in the transition. Further evidence is given by the sudden change in behavior for the $(-)$ metastable branch at $D=D_{2}$. This change can be explained by the fact that the shape of the critical droplet undergoes a drastic change as the relative stabilities of the two metastable states are exchanged. For $D_{2}<D<D_{4}$, the critical droplet is one through which the $(-)$ metastable state decays into the $(0)$ metastable state before it has a chance to decay finally to the (+) stable state. However, for $D_{1}<D<D_{2}$, the (0) metastable state becomes too costly for a system in the $(-)$ metastable state to decay into it. Instead, the critical droplet is one in which the core magnetization is close to that in the $(+)$ equilibrium phase, and the magnetization $m(r)$ passes directly through zero on its way to the value corresponding to the metastable $(-)$ state as $|r| \rightarrow \infty$, without exhibiting a significant plateau corresponding to the (0) metastable state. This droplet is somewhat larger than the one corresponding to an initial decay into the metastable ( 0 ) state, and hence it is more costly to form. We will discuss the implications of this result in Sec. 8.

Based on the work of Langer $[3,5]$, we then conjecture that $\left|\operatorname{Im} f_{\alpha}\right|$ is directly related to the decay rates of the metastable states. In Sec. 6 we will make this connection more quantitative by showing that the scaling behavior of $\left|\operatorname{Im} f_{\alpha}\right|$ agrees with the scaling behavior of the analytically continued free-energy density, considered in Sec. 3.

\section{Finite-Range Scaling of the Constrained Free Energy}

In this section we apply finite-range scaling to the transfer-matrix results of Sec. 5 , and we compare these scaling results to the scaling relations for the analytically continued free energy found in Sec. 3. The results in this section were calculated with $D / J=1.1$ and $T / J=0.25$, corresponding to the $H$-scans shown in Figs. 5 and 6 . We are interested in obtaining values from branches representing a "pure metastable" phase $|\alpha\rangle$, so for each $N$ we select values of $H$ for which $\lambda_{\alpha}=\left(\lambda_{\alpha-1} \lambda_{\alpha+1}\right)^{1 / 2}$, thus ensuring a "safe" distance from the near-degeneracies in the transfer-matrix spectrum. These 


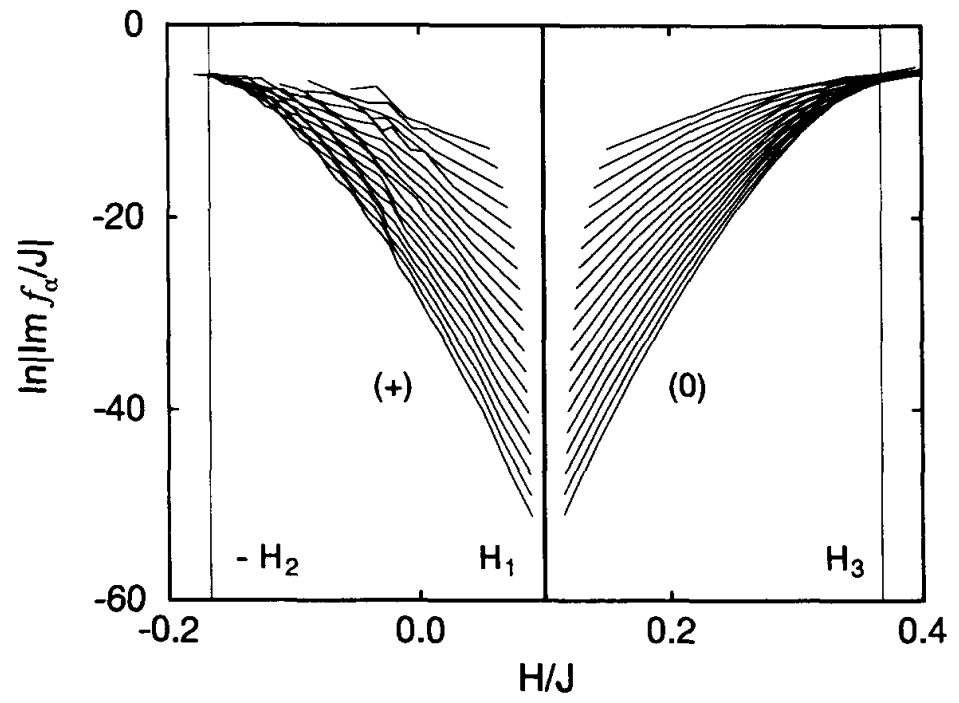

Figure 9: Piecewise-linear envelopes for the lobes of $\ln \left|\operatorname{Im} f_{(+)} / J\right|$ (left) and $\ln \left|\operatorname{Im} f_{(0)} / J\right|$ (right) for a system at $D / J=1.1, T / J=0.25$, and with $N$ in the range $6 \leq N<24$. The transition lines mark the $(+)$-spinodal at $H=-H_{2}$, the first-order transition at $H=H_{1}$, and the (0)-spinodal at $H=H_{3}$. See the text for a detailed description.

points are used to construct envelopes over the lobes of $\left|\operatorname{Im} f_{\alpha}\right|$ shown in Fig. 6. Two sets of such envelopes are shown in Fig. 9, one (left) for the $(+)$ metastable state, and one (right) for the (0) metastable state, for values of $N$ ranging from $N=6$ to $N=24$. As $N$ increases, these values drop exponentially to zero between the firstorder transition at $H_{1}$ and the spinodals at $-H_{2}$ and $H_{3}$, whereas near the spinodals there appears to be a crossover to a slower scaling. This crossover can be seen from the curvature of $\left|\operatorname{Im} f_{\alpha, N}\right|$. As $N$ increases, a singularity in the curvature develops, pushing closer to the spinodal. This singularity was also observed for the Q1DI model [24]. In the present study it was found that some of the branches, particularly for the $(+)$ and $(-)$ metastable states in field regions where they were not the lowest-lying metastable state, showed substantial mixing between nearly degenerate eigenvectors. In these regions the branches did not always extend in such a way as to form envelopes without inflections, even with the above eigenvalue criterion satisfied. An example of this behavior in the (-) metastable state is shown in Fig. 6 for $0<H<H_{2}$. The (+) metastable state shows the same behavior for $-H_{2}<H<0$, which causes the roughness of the envelopes shown in Fig. 9 for this region.

To obtain the scaling behavior for fields inside the spinodal, we first assume a form for $\left|\operatorname{Im} f_{\alpha}\right|$, based on the results of Eqs. (32) and (41), as

$$
\ln \left|\operatorname{Im} f_{\alpha, N}(D, H, T)\right| \sim-\beta N^{\sigma} \Delta(D, H, T),
$$




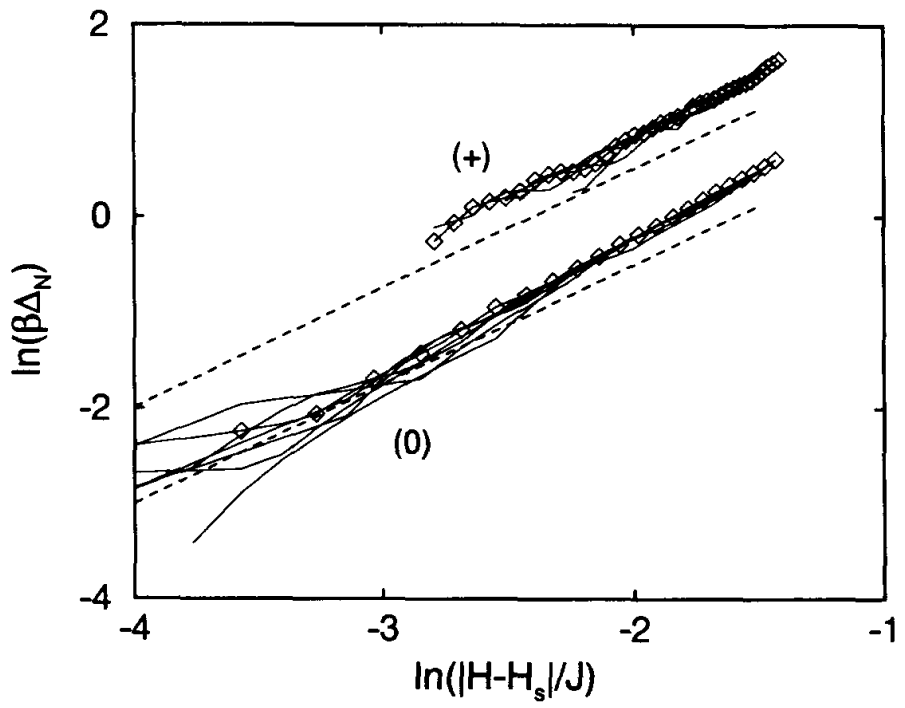

Figure 10: Finite- $N$ estimates for $\beta \Delta(D, H, T)$ in Eq. (58) for the $(0)$ and $(+)$ metastable states, shown on a $\log$-log scale $v s$. $\left|H-H_{s}\right| / J$ for $12 \leq N \leq 24$. The diamonds show the estimates for the largest size, $N=24$. The two dashed lines indicate the corresponding exact $\phi^{3}$ field-theoretical values for $\beta \Delta F$ from Eq. (41). For clarity, both the analytical and numerical results for the $(+)$ state are shifted up by a distance of unity on this logarithmic scale. See the text for a detailed description.

where the exponent $\sigma$ and the function $\Delta(D, H, T)$ are undetermined. By Eq. (32), we expect $N \Delta(D, H, T)$ to be the barrier height $\Delta F$, as given by Eq. (41). Since $\Delta(D, H, T)$ is expected to be independent of $N$, we should find $\sigma=1$. For a given field $H$, we interpolate values of $\ln \left|\operatorname{Im} f_{\alpha, N}\right|$ from the envelope points using a piecewise linear form. A finite-range estimate for $\sigma$ was calculated using methods described in Refs. [24, 43],

$$
\sigma \approx(\ln 2)^{-1} \ln \left(\frac{\ln \left|\operatorname{Im} f_{\alpha, 24}\right|-\ln \left|\operatorname{Im} f_{\alpha, 12}\right|}{\ln \left|\operatorname{Im} f_{\alpha, 12}\right|-\ln \left|\operatorname{Im} f_{\alpha, 6}\right|}\right),
$$

giving $\sigma \approx 1.1$. Finite-range estimates $\beta \Delta_{N}$ for $\beta \Delta(D, H, T)=\beta \Delta F / N$ were then calculated for $12 \leq N \leq 24$, assuming $\sigma=1$ :

$$
\beta \Delta_{N}=-\frac{2}{N}\left(\ln \left|\operatorname{Im} f_{\alpha, N}\right|-\ln \left|\operatorname{Im} f_{\alpha, N / 2}\right|\right) .
$$

Fig. 10 shows the estimates $\beta \Delta_{N}(D, H, T)$ at $D / J=1.1$ and $T / J=0.25$, as calculated by Eq. (60), for the $(0)$ and $(+)$ metastable states respectively, plotted on a $\log -\log$ scale as functions of $\left|H-H_{\mathbf{s}}\right|$. The data for the largest system, $N=24$, are shown as diamonds. These results are compared with the free-energy cost of nucleation obtained from Eq. (41). As can be seen from the figure, the agreement between the extrapolated CTM estimates and the exact results is quite good, considering that the field corrections to the $\phi^{3}$ field theory are expected to be substantial, as was demonstrated in Ref. [24] for the Q1DI model. 


\section{Monte Carlo Results}

The Monte Carlo method is a standard method for studying lattice-gas Hamiltonians, such as the long-range Blume-Capel model. We used the Metropolis algorithm $[49,50,51]$ with non-conserved order parameter, where at each step in the Markov process individual spins were proposed to be flipped at random. The system considered had $L=200$ layers, each containing $N=7$ spins, and periodic boundary conditions were imposed in the $L$-direction. All simulations were performed at $T / J=0.25$, and with a total of 2000 Monte Carlo steps per layer (MCSL). The values of $N, D$, and $H$ were chosen in order to make nucleation reasonably probable in the time allotted.

In Fig. 11(a)-(d) particular realizations of the decay of four metastable states into their respective equilibrium states are shown. Three of their corresponding phase points are shown in Fig. 3 as solid dots. In case (c), the position is not shown in Fig. 3 since the parameters fall outside the displayed region. Horizontally, the spatial direction extends over the $L$ layers, while the vertical axis is the time axis, in units of $10 \mathrm{MCSL}$. The bottom row corresponds to $t=0 \mathrm{MCSL}$, while the top row corresponds to $t=2000 \mathrm{MCSL}$. The gray-scale in the plots relates linearly to the average magnetization within the layer. Black corresponds to $m=+1$, gray to $m=0$, and white to $m=-1$. In Fig. $11(\mathrm{a}), D / J=1.15$ and $H / J=-0.1$. The system starts in the metastable (+) state. After approximately $1000 \mathrm{MCSL}$, a critical droplet of the (0) state forms. The droplet then grows linearly in time by roughly linear motion of the domain walls, until it spans the system. Note the periodic boundary conditions. In Fig. $11(\mathrm{~b}), D / J=0.983$ and $H / J=-0.249$. The system starts in the metastable $(+)$ state. After approximately $200 \mathrm{MCSL}$, a critical droplet forms, and the state decays into the metastable (0) state. The system remains in the metastable $(0)$ state until a critical droplet of the (-) state causes the system to decay into the $(-)$ equilibrium state. In Fig. $11(\mathrm{c}), D / J=0.8$ and $H / J=-0.42$. The free-energy-density functional has two minima, corresponding to a stable $(-)$ state, and a metastable $(+)$ state. In the MC simulation, the system again starts in the metastable $(+)$ state, and we see qualitatively the same behavior, except that the decay goes directly from $(+)$ to $(-)$ since the metastable $(0)$ state does not exist. Note the formation of two independent droplets, which coalesce after they have nucleated. Finally, in Fig. $11(\mathrm{~d}), D / J=0.95$ and $H / J=0.0$ the system is started in the $(0)$ metastable state. Critical droplets of both the $(+)$ and $(-)$ state cause the system to decay. The purely $D$-field-driven domain growth at this phase point, in contrast to the $H$-field-driven growth shown in (a)-(c), appears much more diffuse on the time scale considered in the calculations. After 1000 MCSL the system reaches a configuration where only the $(+)$ and the $(-)$ state are present, reminiscent of late-stage spinodal decomposition. 


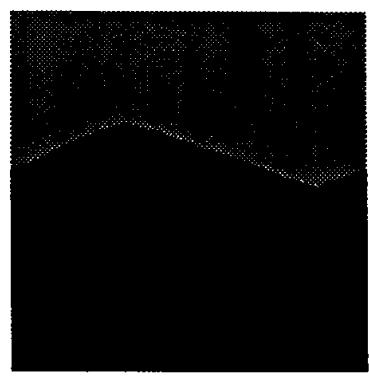

(a)

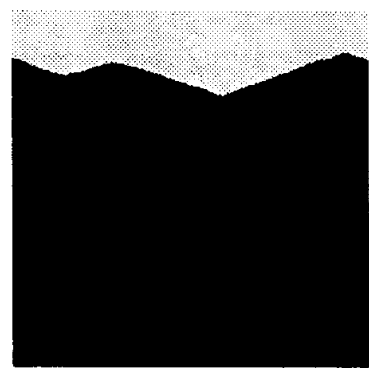

(c)

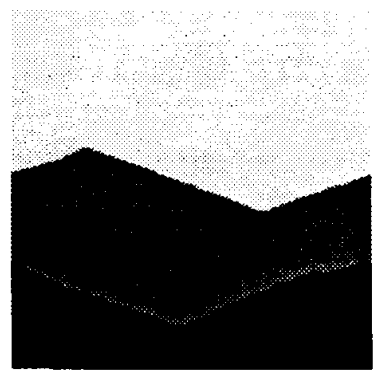

(b)

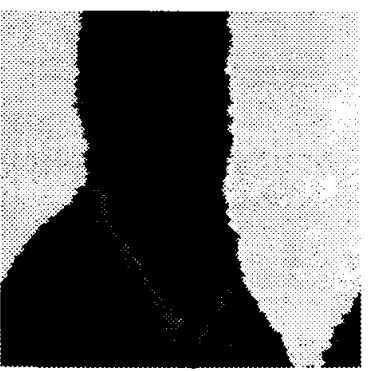

(d)

Figure 11: Dynamical behavior of metastable states in the long-range Blume-Capel model. Simulations with $L=200, N=7$, are performed at $T / J=0.25$, using a total of $t=2000$ Monte Carlo steps per layer. The spatial direction is given horizontally, and the time axis vertically. The gray-scale is chosen so that $m=+1$ is black, $m=0$ gray, and $m=-1$ white. The parameters are: (a) $D / J=1.15, H / J=-0.1$; (b) $D / J=0.983$, $H / J=-0.249 ;$ (c) $D / J=0.8, H / J=-0.42$; and (d) $D / J=0.95, H / J=0$.

\section{Conclusion}

In this paper we have applied analytic continuation and a recently developed constrained-transfer-matrix (CTM) formalism to study the stationary properties of metastability in a system with competing metastable states. In addition we have studied the decay of metastable states by Monte Carlo simulation. Langer $[3,4]$ related the imaginary part of the analytic continuation of the free energy into the region of metastability, $\operatorname{Im} \tilde{F}$, to the decay rate of the metastable states as given by Eq. (1). In a recent study of an Ising model with weak, long-range interactions [24], excellent quantitative agreement was found between the imaginary part of the constrained free-energy density $\operatorname{Im} f_{\alpha}$ and the decay-rate density of the metastable state as measured from the activation barrier. However, as discussed in the introduction, for systems with several metastable states, Gaveau and Schulman [17] have argued that $\operatorname{Im} \tilde{F}$ is not necessarily a valid measure of the decay-rate density. Motivated by this, the purpose of the present work has been to establish to 
what extent the imaginary part of the free-energy density from the CTM formalism can be interpreted as a decay rate in more complicated systems, containing several metastable states.

We have studied a variant of the Blume-Capel model with weak, long-range interactions. One reason for doing so is that for a certain range of fields this model exhibits two competing metastable phases, for which questions can be raised about the interpretation of the analytically continued free energy. Also, the model is simple enough to allow extensive symmetry reduction of the transfer matrix, as shown in Apps. 8 and 8 , so that relatively large system sizes could be considered. We have obtained an exact analytic expression for the finite-temperature spinodal and have used this to calculate the various critical lines in the phase diagram in a very simple manner. In the region of the spinodal we have mapped the Hamiltonian to a $\phi^{3}$ field theory to obtain an expression for the free-energy cost of nucleation. We were thus able to calculate the leading term in an expansion around the spinodal of the "Boltzmann weight" that appears in the analytic continuation of the free energy across the first-order phase transition.

We have outlined the CTM formalism, by which a complex constrained free-energy density can be constructed by reweighting the eigenvalues from the transfer matrix of the equilibrium system. The results for the real part of the metastable constrained free-energy density $\operatorname{Re} f_{\alpha}$ are in excellent agreement with those of the mean-field freeenergy density. The associated imaginary part $\left|\operatorname{Im} f_{\alpha}\right|$ is extremely small, showing exponential dependence on the interaction range inside the classical spinodal. In the region of competing metastability it was found that $\operatorname{Im} f_{\alpha}$ for a metastable state is closely related to the activation barrier involved in the transition, which according to the Van't Hoff-Arrhenius formula of chemical reaction theory, is related to the decay rate. More quantitative evidence was given in Sec. 6, where we applied finite-range scaling to $\left|\operatorname{Im} f_{\alpha}\right|$. There we found very good agreement between the finite-range scaling of $\left|\operatorname{Im} f_{\alpha}\right|$ and the height of the activation barrier obtained by exact analytic continuation.

Our numerical results have demonstrated that the CTM method provides for any pure metastable phase a value for $\left|\operatorname{Im} f_{\alpha}\right|$ that agrees with the Arrhenius law for the decay rate of that particular metastable phase. In the case where two metastable phases are present, the CTM method gives a distinct value of $\left|\operatorname{Im} f_{\alpha}\right|$ for each phase, which can easily be continued through points where these phases are degenerate. The usual procedure of analytic continuation of the free energy, on the other hand, has no mechanism for distinguishing coexistent metastable phases, and thus gives an imaginary part related only to the lowest activation barrier which separates any one of the coexistent phases from the equilibrium phase. An example of this type of result was given in Ref. [17] as a "counterexample" to Eq. (1). We, however, interpret that result as being in agreement with Eq. (1) by noting that the configuration space to which the partition function is constrained, and from which the analytically 
continued free-energy density measures the escape rate, includes both of the degenerate metastable phases, rather than a single pure metastable phase.

In Sec. 7 we studied dynamically the decays of competing metastable states by Monte Carlo simulation. The results confirm that the decay of a particular metastable state might happen either directly or via a succession of separate steps, depending on the availability and relative stability of a second metastable state intermediate between the initial one and the equilibrium phase.

The CTM method has proven to be a highly successful tool in the characterization of metastable phases. It is a nonperturbative method that treats all possible fluctuations in a single calculation. The fluctuations that are important to nucleation are automatically identified, in contrast to the analytical calculations, where the relevant fluctuations must be introduced by hand. This method might therefore be used to study metastability in disordered systems, such as spin glasses, which are known to posses a large number of metastable states so that the critical fluctuations are difficult to characterize. However, the computational requirements of applying the CTM method to such a problem would be enormous.

\section{Acknowledgments}

We would like to thank M. Grant and C. C. A. Günther for useful discussions. T. Fïg was supported by the Danish Research Academy. The research was supported in part by Florida State University through the Supercomputer Computations Research Institute (Department of Energy Contract No. DE-FC05-85ER25000), the Center for Materials Research and Technology, and through Cray Y-MP supercomputer time. This work was also supported by National Science Foundation Grant No. DMR9013107. 


\section{Appendix A. Contraction of the Transfer Matrix}

This appendix provides a mapping of the transfer matrix from layer-configuration space onto a reduced space. Denote the layer-configuration vector space by $\mathcal{X}$. Since the configuration vectors $|X\rangle \in \mathcal{X}$ can be expressed as direct products of single-spin configurations $\left|X_{i}\right\rangle=\left|s_{1, i}\right\rangle\left|s_{2, i}\right\rangle \cdots\left|s_{N, i}\right\rangle, \operatorname{dim}(\mathcal{X})=3^{N}$ for the long-range Blume-Capel model, and the set $\{|X\rangle\}$ constitutes a $3^{N}$-dimensional orthonormal basis for $\mathcal{X}$. The transfer matrix $\mathbf{T}$ is an operator $\mathbf{T}: \mathcal{X} \rightarrow \mathcal{X}$. Assume that we have a symmetry group $G_{U}$ and operators $\mathrm{U}: \mathcal{X} \rightarrow \mathcal{X}$ that represent elements of $G_{U}$ and also commute with $\mathbf{T}$. Symmetry transformation operators must be unitary, so $\mathbf{U}$ must be a cyclic transformation with finite period. This means that the configuration space $\mathcal{X}$ can be divided into equivalence classes, each invariant under $G_{U}$. We denote the equivalence classes by $\mathcal{C}_{k},(k=1, \ldots, K)$, and we relabel the configuration vectors as $\left|X_{k, j}\right\rangle$, where the index $k$ refers to class $\mathcal{C}_{k}$. Thus $\left|X_{k, j}\right\rangle \in \mathcal{C}_{k}$, and the index $j=1, \ldots, g_{k}$ runs through all $g_{k}$ configurations in the class.

Let $O$ be an arbitrary operator that is invariant under $G_{U}$ (for the more general case, in which $O$ has a definite symmetry under $G_{U}$, see Ref. [52]). We then have the following fundamental identity:

$$
\sum_{i=0}^{3^{N}-1} \mathrm{O}\left|X_{i}\right\rangle=\sum_{k=1}^{K} \sum_{j=1}^{g_{k}} \mathrm{O}\left|X_{k, j}\right\rangle .
$$

A reduced vector space $\mathcal{V}$ is spanned by the orthonormal basis $\left\{\left|V_{k}\right\rangle\right\}_{k=1}^{K}$, where

$$
\left|V_{k}\right\rangle=\frac{1}{\sqrt{g_{k}}} \sum_{j=1}^{g_{k}}\left|X_{k, j}\right\rangle
$$

Thus $\operatorname{dim}(\mathcal{V})=K$.

If the layer Hamiltonian for a system is invariant under $G_{U}$, then the transfer matrix $\mathbf{T}$ is also invariant under $G_{U}$. Written as an operator, $\mathbf{T}$ is thus easily transformed into the reduced basis by Eqs. (A1) and (A2):

$$
\begin{aligned}
\mathbf{T} & =\sum_{k, k^{\prime}} \sum_{X \in \mathcal{C}_{k}} \sum_{X^{\prime} \in \mathcal{C}_{k}^{\prime}}|X\rangle e^{-\beta\left\langle X\left|\mathcal{H}^{\circ \mathbf{p}}\right| X^{\prime}\right\rangle}\left\langle X^{\prime}\right| \\
& =\sum_{k, \boldsymbol{k}^{\prime}}\left|V_{k}\right\rangle \sqrt{g_{k} g_{k^{\prime}}} e^{-\beta\left\langle V_{k}\left|\mathcal{H}^{\circ \mathrm{p}}\right| V_{k^{\prime}}\right\rangle}\left\langle V_{k^{\prime}}\right|
\end{aligned}
$$

If we consider the Blume-Capel case, the reduced basis set is $\left|R_{k}\right\rangle \equiv\left|M_{k} Q_{k}\right\rangle$, $k=1, \ldots,(N+1)(N+2) / 2$, and the multiplicity factor $g_{k}=g\left(M_{k}, Q_{k}\right)$ is given by Eq. (6). By substituting appropriately, Eq. (54) is recovered.

Now consider the transformation of Eqs. (47)-(50). Let $|\nu| \in \mathcal{V}$ be an eigenvector in the reduced space, and let $|\alpha\rangle \in \mathcal{X}$ be the corresponding eigenvector in configuration space. It follows from Eq. (A2) that

$$
\left\langle X_{k, j} \mid \alpha\right\rangle=\frac{\left\langle V_{k} \mid \nu\right\rangle}{\sqrt{g_{k}}} \quad(\forall j) .
$$


Expressing $\mathcal{M}_{\alpha}$ in the reduced basis is now simple:

$$
\begin{aligned}
\mathcal{M}_{\alpha} & =\frac{1}{N} \sum_{k=1}^{K} \sum_{j=1}^{g_{k}}\left\langle\alpha \mid X_{k, j}\right\rangle\left\langle X_{k, j}\left|M^{\mathrm{op}}\right| X_{k, j}\right\rangle\left\langle X_{k, j} \mid \alpha\right\rangle \\
& =\frac{1}{N} \sum_{k=1}^{K}\left\langle\nu \mid V_{k}\right\rangle M_{k}\left\langle V_{k} \mid \nu\right\rangle
\end{aligned}
$$

where Eqs. (A1), (A2), and (A4) are used, and the multiplicity factors introduced by Eq. (A4) are canceled in the sum. The transformation of Eqs. (47)-(50) are all obtained in the same manner. 


\section{Appendix B. Decomposition of the transfer matrix}

In this appendix the transfer matrix $\mathbf{T}$ is decomposed by applying a unitary transformation $\mathbf{S}$ so that $\mathbf{S T S}^{t}$ is block diagonal, which allows the separation of all the zero eigenvalues of $T$. The dimension of the original transfer matrix from App. 8 is $\operatorname{dim}(\mathbf{T})=(N+1)(N+2) / 2$, while the reduced transfer matrix has the dimension of $\operatorname{rank}(\mathrm{T})=2 N+1$.

The transfer matrix can be written as $\mathbf{T}=\mathbf{D A D}$, where $\mathbf{D}$ is a diagonal matrix that contains interactions within a layer, and $\mathbf{A}$ contains interactions between layers [53]. In the Blume-Capel case the matrix elements of $\mathbf{A}$ are given by

$$
\left\langle M Q|\mathbf{A}| M^{\prime} Q^{\prime}\right\rangle=\exp \left[\beta J M M^{\prime} / N\right],
$$

and the matrix elements of $\mathbf{D}$ are given by

$$
\left\langle M Q|\mathbf{D}| M^{\prime} Q^{\prime}\right\rangle=\sqrt{g(M, Q)} \exp [-\beta(D Q-H M) / 2] \delta_{M, M^{\prime}} \delta_{Q, Q^{\prime}}
$$

The matrix $\mathbf{A}$ is independent of $Q$ and $Q^{\prime}$, which means that the rank of $\mathbf{A}$ is just the number of distinct values of the magnetization, since $\mathbf{D}$ is of full rank; $\operatorname{rank}(\mathrm{T})=\operatorname{rank}(\mathrm{A})=2 N+1$. We organize the basis vectors $|M Q\rangle$ in groups according to the magnetization $M,(M=-N,-N+1, \ldots, N)$. As an example, the basis vectors for $N=2$ are organized in the following way: $\{|-22\rangle,|-11\rangle,|00\rangle,|02\rangle$, $|11\rangle,|22\rangle\}$. It can be shown in general for the long-range Blume-Capel model, that the number of basis vectors $n(M)$ with magnetization $M$ is $n(M)=\lfloor 1+(N-|M|) / 2\rfloor$, where $[\cdots]$ denotes the integer value.

We start by constructing a matrix $\mathbf{B}$ that block diagonalizes $\mathbf{A}$. Let $\mathbf{B}$ be a block diagonal matrix, where the $n(M) \times n(M)$ square block corresponding to magnetization $M$ is denoted $\mathbf{B}_{M}$, and define an $n(M) \times n(M)$ square matrix $\mathbf{J}_{M}$ with all its elements equal to unity. We define $\mathbf{B}_{M}$ as a unitary matrix that diagonalizes $\mathbf{J}_{M}$. This will be satisfied if we choose the column vectors of $\mathbf{B}_{M}$ to be an orthonormal basis of eigenvectors for $\mathbf{J}_{M}$. However, $\mathbf{B}_{M}$ is not uniquely defined in this fashion, since $\operatorname{rank}\left(\mathbf{J}_{M}\right)=1$. To make a specific choice for $\mathbf{B}_{M}$, we select the eigenvector corresponding to the single nonzero eigenvalue of $\mathbf{J}_{M}$ as the last column.

We define a matrix $\mathbf{C}$ by $\mathbf{C} \equiv \mathbf{B A B}^{\mathfrak{t}}$. Due to the particular choice for all $\mathbf{B}_{M}$, it can be shown with a little effort that the $\mathbf{C}$ matrix has a special matrix form, which 
we will call a C-form:

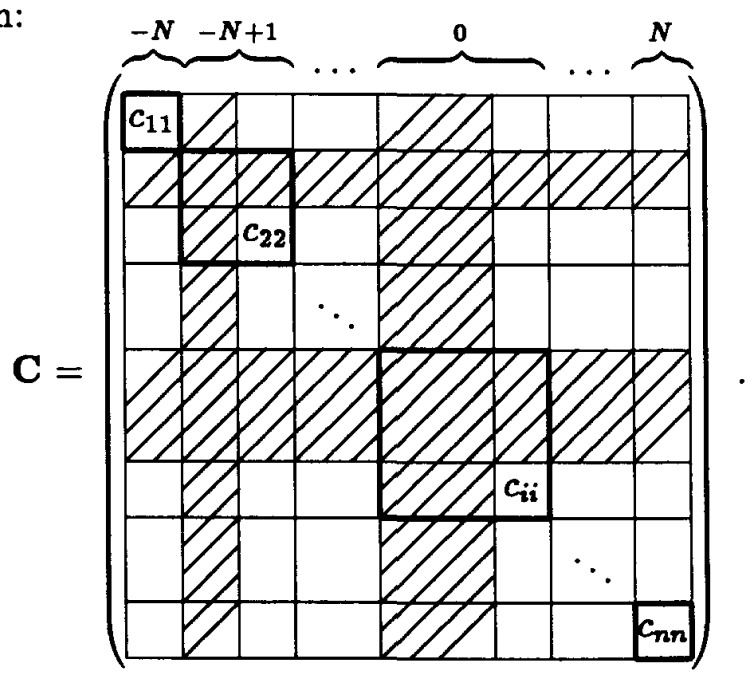

As indicated by the curly brackets in Eq. (B3) the basis vectors are grouped together according to magnetization $M=-N,-N+1, \ldots, N$. All elements within the hashed regions are zero, and the only nonzero elements occupy the last row and column within each magnetization group. From Eq. (B3) it is clear that there exists a permutation matrix $\mathbf{P}$ such that $\mathbf{P C P}^{\mathfrak{t}}$ reduces to a block matrix of size $(2 N+1) \times(2 N+1)$, and all elements outside this block are zero. For each diagonal block $\mathbf{C}_{M}$ (outlined by thick lines in Eq. (B3)), with $\operatorname{dim}\left(\mathbf{C}_{M}\right)=n(M)$, there is only one nonzero element, which is located at the bottom row diagonal position.

The unitary transformation matrix $\mathbf{S}$ that block diagonalizes $\mathbf{T}$ can now be determined based on the properties of the C-form. The transformation of $\mathbf{T}$ can be expanded as

$$
\mathbf{S T S}^{\mathbf{t}}=\left(\mathbf{S D B}^{\mathrm{t}}\right)\left(\mathbf{B A B}^{\mathrm{t}}\right)\left(\mathbf{S D B}^{\mathrm{t}}\right)^{\mathrm{t}}
$$

Hence from Eq. (B4) it follows that the transformed transfer matrix STS ${ }^{t}$ will have the C-form if the matrix product between $\mathbf{S D B}^{\mathbf{t}}$ and $\mathbf{C}$ preserves the C-form. It is sufficient to restrict the unitary transformation $\mathbf{S}$ so that it can be decomposed into a block diagonal matrix. The blocks are denoted $\mathbf{S}_{M},(M=-N,-N+1, \ldots, N)$.

Therefore SDB $^{t}$ also decomposes into a block diagonal matrix, and it suffices to show that for all $M, \mathbf{S}_{M}\left(\mathbf{D}_{M} \mathbf{B}_{M}^{t}\right) \mathbf{C}_{M}$ has the same form as $\mathbf{C}_{M}$. This is equivalent to requiring that all rows of $S_{M}$, except for the last row, are orthogonal to the last column in the matrix product $\mathbf{D}_{M} \mathbf{B}_{M}^{t}$. Since all columns in $\mathbf{D}_{M} \mathbf{B}_{M}^{t}$ are linearly independent, $\mathbf{S}_{M}$ can be constructed by using a Gram-Schmidt orthogonalization process on the column vectors in $\mathbf{D}_{M} \mathbf{B}_{M}^{t}$. Since $\mathbf{S}$ is unitary, the transformed transfer matrix $\mathbf{S T S} \mathbf{S}^{\mathrm{t}}$ is symmetric and can be reduced by the permutation matrix $P$ into a $(2 N+1) \times(2 N+1)$ reduced transfer matrix. This concludes the proof of the decomposition. 


\section{References}

[1] J. D. Gunton, M. San Miguel, and P. S. Sahni, in Phase Transitions and Critical Phenomena, Vol. 8, edited by C. Domb and J. L. Lebowitz (Academic, London, 1983).

[2] K. Binder, Rep. Prog. Phys. 50, 783 (1987).

[3] J. S. Langer, Ann. Phys. 41, 108 (1967).

[4] J. S. Langer, Phys. Rev. Lett. 21, 973 (1968).

[5] J. S. Langer, Ann. Phys. 54, 258 (1969).

[6] K. Binder and E. Stoll, Phys. Rev. Lett. 31, 47 (1973).

[7] K. Binder, Phys. Rev. B 8, 3423 (1973).

[8] K. Binder and H. Müller-Krumbhaar, Phys. Rev. B 9, 2328 (1974).

[9] R. J. McCraw and L. S. Schulman, J. Stat. Phys. 18, 293 (1978).

[10] R. J. McCraw, Phys. Lett. 75A, 379 (1980).

[11] C. M. Newman and L. S. Schulman, J. Stat. Phys. 23, 131 (1980).

[12] G. Roepstorff and L. S. Schulman, J. Stat. Phys. 34, 35 (1984).

[13] M. Büttiker and R. Landauer, Phys. Rev. Lett. 43, 1453 (1979).

[14] M. Büttiker and R. Landauer, Phys. Rev. A 23, 1397 (1981).

[15] W. Klein and C. Unger, Phys. Rev. B 28, 445 (1983).

[16] C. Unger and W. Klein, Phys. Rev. B 29, 2698 (1984).

[17] B. Gaveau and L. S. Schulman, Lett. Math. Phys. 18, 201 (1989).

[18] V. Privman and L. S. Schulman, J. Phys. A 15, L231 (1982).

[19] V. Privman and L. S. Schulman, J. Stat. Phys. 31, 205 (1982).

[20] P. A. Rikvold, Prog. Theor. Phys. Suppl. 99, 95 (1989).

[21] P. A. Rikvold, Physica Scripta T38, 36 (1991).

[22] P. A. Rikvold, B. M. Gorman, and M. A. Novotny, AIP Conf. Proc. 256, 549 (1992).

[23] M. A. Novotny, W. Klein, and P. A. Rikvold, Phys. Rev. B 33, 7729 (1986). 
[24] B. M. Gorman, P. A. Rikvold, and M. A. Novotny, preprint FSU-SCRI-93-153, submitted to Phys. Rev. E.

[25] C. C. A. Günther, P. A. Rikvold, and M. A. Novotny, Phys. Rev. Lett. 71, 3898 (1993).

[26] C. C. A. Günther, P. A. Rikvold, and M. A. Novotny, (unpublished).

[27] P. A. Rikvold, H. Tomita, S. Miyashita, and S. W. Sides, (unpublished).

[28] M. E. Fisher, Physics 3, 255 (1967).

[29] O. Penrose, Heriot-Watt University preprint (1993).

[30] P. Hänggi, P. Talkner, and M. Borkovec, Rev. Mod. Phys. 62, 251 (1990).

[31] M. Blume, Phys. Rev. 141, 517 (1966).

[32] H. W. Capel, Physica 32, 966 (1966).

[33] M. Blume, V. J. Emery, and R. B. Griffiths, Phys. Rev. A 4, 1071 (1971).

[34] P. A. Rikvold, Electrochim. Acta 36, 1689 (1991), and refs. cited therein.

[35] A. N. Berker and M. Wortis, Phys. Rev. B 14, 4946 (1976).

[36] J. D. Kimel, P. A. Rikvold, and Y.-L. Wang, Phys. Rev. B 45, 7237 (1992).

[37] D. P. Landau, Phys. Rev. B 33, 7700 (1986).

[38] R. J. Speedy and C. A. Angell, J. Chem. Phys. 65, 851 (1976).

[39] A. J. Leggett, Rev. Mod. Phys. 47, 331 (1975).

[40] J. R. Schrieffer, Theory of Superconductivity (Addison-Wesley, New York, 1983).

[41] K. Binder, Phys. Rev. A 29, 341 (1984).

[42] R. B. Griffiths, Phys. Rev. Lett. 13, 715 (1970).

[43] P. A. Rikvold, B. M. Gorman, and M. A. Novotny, Phys. Rev. E 47, 1474 (1993).

[44] W. Paul, D. W. Heermann, and K. Binder, J. Phys. A 22, 3325 (1989).

[45] C. Domb, Adv. Phys. 9, 149 (1960).

[46] M. Marcus and H. Minc, A Survey of Matrix Theory and Matrix Inequalities (Dover, New York, 1964). 
[47] R. E. Blahut, Principles and Practice of Information Theory (Addison-Wesley, Reading, 1987), pp. 61-64.

[48] W. H. Press, S. A. Teukolsky, W. T. Vetterling, and B. P. Flannery, Numerical Recipes in $C$ (Cambridge University Press, Cambridge, 1992).

[49] D. W. Heermann and A. N. Burkitt, Parallel Algorithms in Computational Science (Springer, Berlin, 1991).

[50] K. Binder, in Monte Carlo Methods in Statistical Physics, edited by K. Binder (Springer, Berlin, 1979).

[51] K. Binder and D. W. Heermann, Monte Carlo Simulation in Statistical Physics (Springer, Berlin, 1988).

[52] P. A. Rikvold, K. Kaski, J. D. Gunton, and M. C. Yalabik, Phys. Rev. B 29, 6285 (1984).

[53] M. A. Novotny, J. Math. Phys. 20, 1146 (1979). 


\section{DISCLAIMER}

This report was prepared as an account of work sponsored in part by an agency of the United States Government. Neither the United States Government nor any agency thereof, nor any of their employees, makes any warranty, express or implied, or asumes any legal liability or responsibility for the accuracy, completeness, or usefulness of any information, epparatus, product, or process disclosed, or represents that its use would not infringe privately owned rights. Reforence herein to any specific commercial product, process, or service by trade name, trademark, manufacturer, or otherwise, does not necessarily constitute or imply its endorsement, recommendation, or favoring by the United States Government or any agency thereof. The views and opinions of authors expressed herein do not necessarily state or reflect those of the United States Government or any agency thereof. 